\title{
Analysis of a Local Hydrodynamic Model with Marangoni Effect
}

\author{
J. Monnier \& P. Witomski \\ Laboratoire de Modelisation et Calcul (LMC-IMAG), BP 53, F-38041 \\ Grenoble Cedex 9, France. \\ Projet IDOPT (CNRS-INPG-INRIA-UJF)
}

\begin{abstract}
We study a mathematical model treating of the dynamic contact line problem, supposed to describe the main features of the advancing triple line (rolling motion and variable contact angle) and to remove the singularity. The model is composed by a macroscopic hydrodynamic free surface flow model (Navier-Stokes) coupled with a mesoscopic local surface model. Detailed mathematical and numerical analysis of the 1D steady-state local surface model are done: existence and uniqueness of the exact and numerical solutions, extra properties of the derivatives, and convergence of finite element schemes. Some numerical results of the two models treated separately are presented for a $2 \mathrm{D}$ plunging tape configuration.
\end{abstract}

\section{Introduction}

We study a mathematical model treating of the dynamic contact line problem: the motion of an advancing liquid on a solid surface and displacing a gas. Our final goal is to simulate numerically the motion of a such flow. The present paper is the first part of our study.

The physical phenomena of dynamical contact line appears in many industrial processes such as coating of solids by liquids.

Two main features of such viscous and slow flows revealed by experiments are the following:

i) the liquid front advances following a rolling motion, similar to a caterpillar vehicle, see [5]: the particles of the liquid-gas interface arrive at the solid-liquid interface.

ii) the dynamical contact angle derives from its static value -determined 
by the classical Young equation-, and depends on the fluid velocity in the bulk. In addition, it seems that its value cannot be prescribed explicitly in a general way, see [1] and the references cited therein for a more complete review.

The contact angle and the triple line velocity are ones of the most important parameter to describe the motion of such flows.

The mathematical modeling of the moving contact line is delicate. A noslip boundary condition at the solid-liquid interface implies a non-physical singularity: the fluid exerts an infinite force on the solid surface, [5].

Then, most of the theories and most of models have been based on a slippage description, see e.g. [8], [6], [3], see also [4], [9] for a more complete review.

A slip condition removes the singularity, however, it replaces the rolling motion by a sliding one, [6], [10]. For a normal liquid flowing over a smooth solid, slippage is usually negligible, [4].

The mathematical model studied in the present paper is the Shikhmurzaev's model established and presented in [9], [10], [11], [1]. This model is supposed to describe the main features of the advancing contact line and to remove the singularity.

The main idea of this model is the following. The rolling motion induces a local variation of the surface tension. This variation would be due to fluid particles going from the liquid-gas interface to the liquid-solid one. The surface tension gradient induced, influences the motion and the force near the contact line, and implies a Marangoni effect. In this model, the (dynamic) wetting angle is not imposed but is a response of the model.

In a mathematical point of view, the Shikhmurzaev's model is composed by a macroscopic Hydrodynamic Free Surface Model -HFSM- (NavierStokes incompressible) fully coupled with a mesoscopic Local Surface Model -LSM- (non linear degenerated time-dependent equation).

The paper is organized as follows. In section 2, we present the Shikhmurzaev's model established in [9], [10], [11], [1] and we reformulate its equations. In section 3 , we study mathematically the $1 \mathrm{D}$ steady-state version of the LSM. In Section 3.1, the existence and uniqueness of the solution is proved, and extra properties on its derivatives are stated. In section 3.2, we discretize the LSM using the finite element method, and we prove the existence, the local uniqueness and the convergence of the finite element solution. In section 4, numerical results are presented. First, we consider the 1D LSM and present some numerical tests. Then, we consider independently a sim- 
plified version of the HFSM with the local Marangoni term given. Some simulations corresponding to a plunging tape configuration are performed. The complete model will be studied in the second part of the present article. The numerical schemes, algorithms and numerical results obtained will be presented therein.

\section{The Mathematical Model}

The model studied in the present paper and presented below derives from [9], [10], [11] and [1].

Let $\Omega$ be a liquid drop $(L)$ wetting a solid $(S)$. We denote by $\Gamma_{S L}$ the solid-liquid contact surface, by $\Gamma_{S G}$ the solid-gas contact surface, by $\Gamma_{L G}$ the free surface liquid-gas and by $\gamma_{C}$ the contact line liquid-gas-solid (the triple line).

When the liquid is at rest, the (static) contact angle $\theta_{s}$ satisfies the classical Young equation:

$$
\sigma_{L G}^{e q} \cos \left(\theta_{s}\right)=\sigma_{S G}^{e q}-\sigma_{S L}^{e q}
$$

where $\sigma_{L G}^{e q}, \sigma_{S L}^{e q}$ and $\sigma_{S G}^{e q}$ are the equilibrium surface tensions of the liquidgas, solid-liquid and solid-gas interfaces respectively.

The interface fluid-gas is considered at a macroscopic length scale and the solid surface is supposed to be perfectly smooth.

When the drop is moving, for example when sliding on an inclined plane, the contact angle and the drop shape are variable. The aim of the present study is to simulate numerically a such phenomena using the Shikhmurzaev's model, [9, 1].

A macroscopic Hydrodynamic Free Surface Model -HFSM-, for the fluid motion is coupled to a mesoscopic Local Surface Model -LSM- describing the surface tension distribution and the contact line motion. The HFSM consists to the Navier-Stokes equations with free surface and original boundary conditions. The coupling with the LSM is done through these boundary conditions imposed on a small vicinity of the triple line. The LSM describes the dependence between the surface tension parameters and the fluid motion.

Let us give the equations of the full model in two dimensions of space. Figures 2 and 2 illustrate the model for the advancing angle of a droplet wetting partially a solid. 


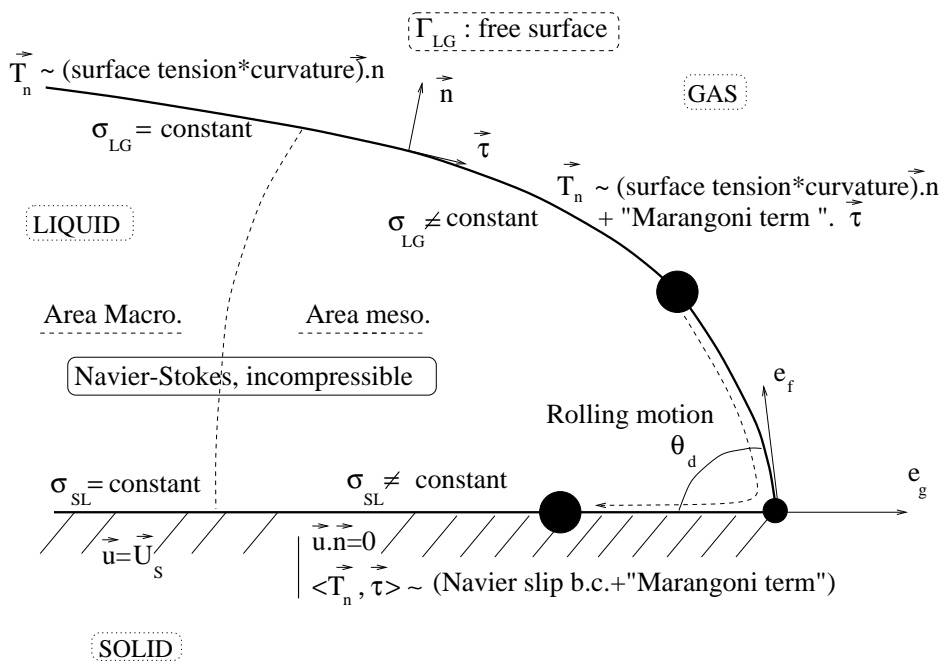

Figure 1: Liquid droplet wetting a solid surface. The advancing angle.

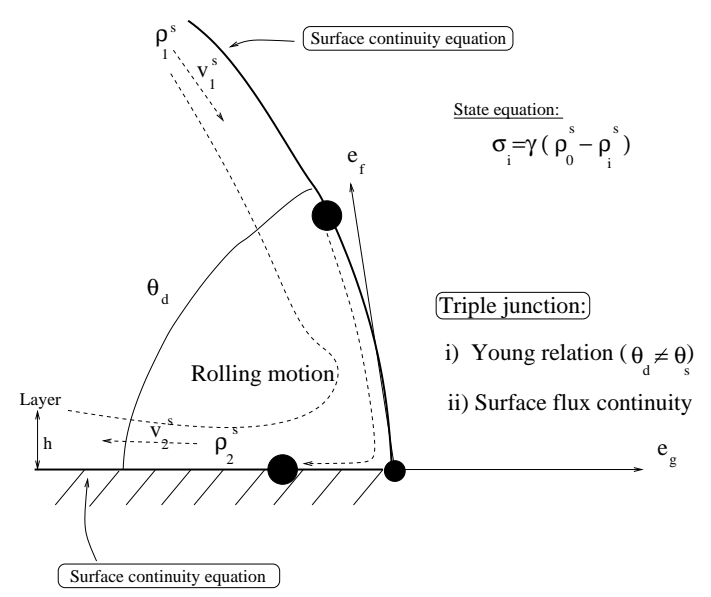

Figure 2: Vicinity of the triple junction -the mesoscopic area-. 


\subsection{The 2D macroscopic Hydrodynamic Free Surface Model}

We denote by $\vec{u}$ the fluid velocity, $p$ its pressure, $\Sigma$ the stress tensor,

$$
\Sigma_{i j}=-p \delta_{i j}+\mu\left(\partial_{i} u_{j}+\partial_{j} u_{i}\right) \quad 1 \leq i, j \leq 2
$$

where $\mu$ is the dynamic viscosity.

We denote by $(\vec{\tau}, \vec{n})$ the unit tangential and external normal vectors such that it is direct. We set:

$$
\overrightarrow{\Sigma_{n}}=\Sigma \cdot \vec{n} \in \mathbb{R}^{2} ; \quad \overrightarrow{\Sigma_{n}}=\Sigma_{n} \vec{n}+\Sigma_{\tau} \vec{\tau}
$$

The fluid motion is governed by the incompressible Navier-Stokes equations:

$$
\left\{\begin{array}{rlcc}
\hat{\rho} \frac{\partial u_{i}}{\partial t}-(\operatorname{div}(\Sigma))_{i}+\hat{\rho}(\vec{u} . \vec{\nabla}) u_{i} & =\hat{\rho} g_{i} & & \text { in }(0, T) \times \Omega \quad 1 \leq i \leq 2 \\
\operatorname{div}(\vec{u}) & = & 0 & \text { in }(0, T) \times \Omega
\end{array}\right.
$$

where $\hat{\rho}$ is the fluid density, $\vec{g}$ the gravity, $T$ the final time and

$$
(\operatorname{div}(\Sigma))_{i}=\sum_{j=1}^{2} \partial_{j} \Sigma_{i j}
$$

To describe the boundary conditions, we decompose $\Gamma_{S L}$ (respectively $\Gamma_{L G}$ ) in two parts $\Gamma_{S L}^{M}$ and $\Gamma_{S L}^{m}$ (respectively $\Gamma_{L G}^{M}$ and $\Gamma_{L G}^{m}$ ). The notation $M$ (respectively ${ }^{m}$ ) refers to the macroscopic (respectively mesoscopic) boundary -see Fig. 2-.

The boundary conditions on the free surface (liquid-gas) are

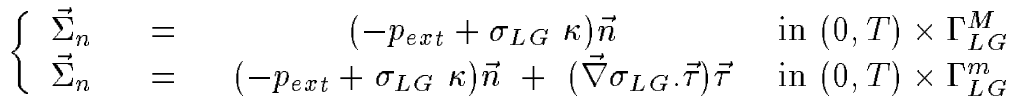

where $\kappa$ is the mean curvature and $p_{e x t}$ is the external pressure.

The liquid-solid contact is described by

$$
\vec{u}=\vec{U}_{S} \quad \text { in }(0, T) \times \Gamma_{S L}^{M}
$$

where $\vec{U}_{S}$ is the solid velocity, and

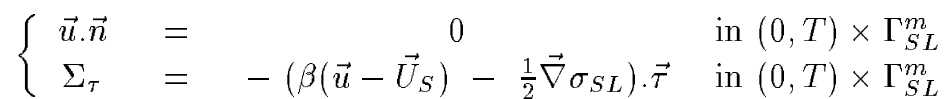


where $\beta>0$ is a sliding type coefficient. We have $\beta \approx \frac{\mu}{h}$ where $h$ is the layer thickness, see [9] and Fig. 2.

Let us point out that in the Shikhmurzaev's model, there is no actual slip between the fluid and the solid surface, [9]. The boundary condition (7) models an apparent slip, where the surface velocity $v_{i}^{s}$ introduced below -see relations (15), (16) and Fig. 2- models a mean velocity in the layer of thickness $h$.

In other respect, the boundary condition (7) removes the shear-stress singularity.

Surface tension gradients appear in (5) and (7). It is a particularity of the present model. Let us recall that surface tension gradients imply a flow towards the region of higher surface tension (the Marangoni effect). In the present model, it is a self-induced Marangoni effect. As a matter of fact, the Shikhmurzaev's model is based on the assumption that the rolling motion, described in [5], implies a mechanical and local change of the surface tension coefficients, see [9].

Remark 2.1 Far enough from the triple point i.e. in the so-called macroscopic area, one had assumed that the fluid sticks to the solid (no-slip boundary conditions) and that the surface tension gradient $\vec{\nabla} \sigma_{L G}$ vanishes. In others words, the surface tension gradients are supposed to be non null only in the so-called mesoscopic area $\Gamma_{S L}^{m}$ and $\Gamma_{L G}^{m}$.

Remark 2.2 If $\vec{\nabla} \sigma_{S L}=\vec{\nabla} \sigma_{L G}=0$ then the boundary conditions are more classical. In that case, the boundary condition (7) is a Navier slip type boundary condition.

The free surface dynamic We define the free surface $\Gamma_{L G}$ as the graph of a function $\varphi\left(t, x_{1}\right)$,

$$
\Gamma_{L G}=\left\{x_{2}, x_{2}=\varphi\left(t, x_{1}\right), x_{1} \in I_{x}, t \in(0, T)\right\}
$$

Then, the free surface motion is described by the following equation:

$$
\frac{\partial \varphi}{\partial t}+u_{1} \frac{\partial \varphi}{\partial x_{1}}=-u_{2} \quad \text { in }(0, T) \times I_{x}
$$

with initial conditions given. The boundary conditions is:

$$
\varphi(t, \hat{x}) \quad \text { given }
$$

if the extremity point $\hat{x}$ of the surface is an inflow extremity. 
Using the representation (8) of $\Gamma_{L G}$, we have the expression of the mean curvature:

$$
\kappa\left(t, x_{1}\right)=\frac{\partial}{\partial x_{1}}\left(\frac{\frac{\partial \varphi}{\partial x_{1}}}{\sqrt{1+\left|\frac{\partial \varphi}{\partial x_{1}}\right|^{2}}}\right)\left(t, x_{1}\right) \quad \text { in }(0, T) \times I_{x}
$$

Remark 2.3 Let us point out an important feature of the model. The dynamic wetting angle $\theta_{d}$ is not imposed. It is a response of the model. It can be computed using the relation:

$$
\operatorname{cotan}\left(\theta_{d}\right)=-\frac{\partial \varphi}{\partial x_{1}}\left(t, P_{C}\right) \text { in }(0, T)
$$

where $P_{C}$ is the triple point liquid-solid-gas.

If $\sigma_{L G}, \vec{\nabla} \sigma_{L G}$ and $\vec{\nabla} \sigma_{S L}$ are given in the mesoscopic areas $\Gamma_{S L}^{m}$ and $\Gamma_{L G}^{m}$, then the system (4)-(10) models a free surface Navier-Stokes flow with surface tension, with a local sliding type boundary condition taking account a Marangoni effect. With initial conditions, this system is closed. In all the sequel, we omit the $\overrightarrow{ }$ on the vectorial quantities.

\subsection{The mesoscopic Local Surface Model}

In the Shikhmurzaev's model [9], the Young equation is supposed to remain valid when the contact line is moving:

$$
\sigma_{L G} \cos \left(\theta_{d}\right)=\sigma_{S G}-\sigma_{S L}
$$

Since the dynamic angle $\theta_{d}$ is different from its static value $\theta_{s}$ when the contact line is moving, this assumption implies that at least one surface tension coefficient $\sigma$ changes from its equilibrium value $\sigma^{e q}$.

The Shikhmurzaev's model is based on the assumption that the rolling motion [5] implies a local change of the surface tension coefficients from its equilibrium value to a different value, in a finite time $\tau^{*}$ called the relaxation time, see [9].

In others respects, it follows from the experiments presented in [1] that the surface tension coefficients depend locally on the contact line velocity and on the fluid flow in the bulk. So, the macroscopic HFSM and the mesoscopic LSM are fully coupled.

Now, let us present the so-called mesoscopic LSM as it is established in $[9]$. 
Roughly, the interfaces are described by surface densities $\rho^{s}$. These surface densities are solution of surface continuity equations. A state equation gives the relation between $\rho^{s}$ and the surface tension coefficients $\sigma$.

We denote by $\rho_{i}^{s}, i=1,2$, the surface density on $\Gamma_{L G}(\mathrm{i}=1)$ and on $\Gamma_{S L}(\mathrm{i}=2)$. The surface tension is related to the excess density through a linear state equation

$$
\sigma_{i}=\gamma\left(\rho_{0}^{s}-\rho_{i}^{s}\right) \quad i=1,2
$$

where $\gamma$ and $\rho_{0}^{s}$ are given constants.

We have the surface continuity equation:

$$
\frac{\partial \rho_{i}^{s}}{\partial t}+\operatorname{div}\left(\rho_{i}^{s} v_{i}^{s}\right)+\frac{1}{\tau^{*}}\left(\rho_{i}^{s}-\rho_{i}^{e q}\right)=0 \quad i=1,2
$$

where $\tau^{*}$ is the relaxation time relative to the rolling motion, $v_{i}^{s}$ is a mean velocity inside the layer and $\rho_{i}^{e q}$ is its density at equilibrium, [9]: $\sigma_{i}\left(\rho_{i}^{e q}\right)=\sigma_{i}^{e q}, i=1,2$.

The velocity $v_{1}^{s}$ (respectively $v_{2}^{s}$ ) is related to $\rho_{1}^{s}$ (respectively $\rho_{2}^{s}$ ) and to the fluid velocity $u$ (respectively the solid velocity $U_{S}$ ). We have the following Darcy laws type, [9]:

$$
\begin{gathered}
\left(1+4 \alpha_{1} \alpha_{2}\right) \nabla \sigma_{L G}=4 \alpha_{2}\left(v_{1}^{s}-u\right) \\
v_{2}^{s}=\alpha_{1} \nabla \sigma_{S L}+\frac{1}{2}\left(u+U_{S}\right)
\end{gathered}
$$

where $\alpha_{i}, i=1,2$, are given constants characterizing the viscous properties of the interface.

Remark 2.4 The macroscopic parts $\Gamma_{S L}^{M}$ and $\Gamma_{L G}^{M}$, it follows from (15)(16) and (6) that:

$$
\begin{gathered}
v_{1}^{s}=u \quad \text { in }(0, T) \times \Gamma_{L G}^{M} \\
v_{2}^{s}=u=U_{S} \quad \text { in }(0, T) \times \Gamma_{S L}^{M}
\end{gathered}
$$

In addition, one has:

$$
v_{1}^{s} . n=u . n \quad \text { in }(0, T) \times \Gamma_{L G}^{m}
$$

At the triple junction, the surface flux continuity is imposed:

$$
\left(\rho_{1}^{s} v_{1}^{s}\right) e_{f}=\left(\rho_{2}^{s} v_{2}^{s}\right) e_{g}
$$


where $e_{f}$ and $e_{g}$ are unit vectors normal to the contact line and tangential to the gas-liquid and gas-solid interface respectively, Fig. 2.

Let us notice that: $\cos \left(\theta_{d}\right)=-e_{f} \cdot e_{g}$.

The system (4)-(17) with given initial conditions is closed.

\section{Reformulation of the LSM}

We set: $\xi=\gamma \frac{\left(1+4 \alpha_{1} \alpha_{2}\right)}{4 \alpha_{2}}$. It follows from (13) with $i=1$ and (15) that:

$$
v_{1}^{s}=u-\xi \nabla \rho_{1}^{s}
$$

Then, the mesoscopic LSM on the liquid-gas interface becomes:

$$
\left\{\begin{array}{rll}
\frac{\partial \rho_{1}^{s}}{\partial t}-\xi \operatorname{div}\left(\rho_{1}^{s} \nabla \rho_{1}^{s}\right)+\operatorname{div}\left(\rho_{1}^{s} u\right)+\frac{1}{\tau^{*}} \rho_{1}^{s} & =\frac{1}{\tau^{*}} \rho_{1}^{e q} & \text { in }(0, T) \times \Gamma_{L G}^{m} \\
\rho_{1}^{s} & =\rho_{1}^{e q} & \text { on }(0, T) \times J_{L G} \\
\left(\rho_{1}^{s}\left(u-\xi \nabla \rho_{1}^{s}\right)\right) & \text { given } & \text { on }(0, T) \times P_{C}
\end{array}\right.
$$

where $J_{L G}$ is the junction point of $\Gamma_{L G}^{m}$ and $\Gamma_{L G}^{M}$, and $P_{C}$ is the triple point.

With initial conditions, the LSM on the free surface liquid-gas (19) is closed.

Let us treat the case $i=2$. We set $\lambda=\alpha_{1} \gamma$ and:

$$
U=\frac{1}{2}\left(u+U_{S}\right)
$$

Using (13) with $i=1$ and (16), we obtain:

$$
v_{2}^{s}=U-\lambda \nabla \rho_{2}^{s}
$$

Then, we obtain the mesoscopic LSM on the solid-liquid interface:

$$
\left\{\begin{array}{rll}
\frac{\partial \rho_{2}^{s}}{\partial t}-\lambda \operatorname{div}\left(\rho_{2}^{s} \nabla \rho_{2}^{s}\right)+\operatorname{div}\left(\rho_{2}^{s} U\right)+\frac{1}{\tau^{*}} \rho_{2}^{s} & =\frac{1}{\tau_{e}^{*}} \rho_{2}^{e q} & \text { in }(0, T) \times \Gamma_{S L}^{m} \\
& =\rho_{2}^{e q} & \text { on }(0, T) \times J_{L G} \\
\left(\rho_{2}^{s}\left(-\lambda \nabla \rho_{2}^{s}+U\right)\right) & \text { given } & \text { on }(0, T) \times P_{C}
\end{array}\right.
$$

where $J_{S L}$ is the junction point of $\Gamma_{S L}^{m}$ and $\Gamma_{S L}^{M}$.

With initial conditions, the LSM on the solid-liquid surface (22) is closed. 
The two models (19) and (22) are very similar. In a mathematical point of view, it is non linear degenerated unsteady equations.

The numerical simulation of the complete model (HFSM fully coupled with LSF) will be treated in an other article (part II).

The purpose of next section is to give mathematical and numerical analysis of the 1D mesoscopic LSM. Then, we present some 1D and 2D numerical results as applied to a plunging tape configuration. The goal being to understand the LSM contribution to the bulk fluid motion.

\section{The 1D Local Surface Model}

We study the 1D steady-state version of the Local Surface Model -LSM-. First, we detail the mathematical analysis. Under some physical assumptions, we prove the existence, the uniqueness of the weak solution and some useful estimates of the solution, the first and second derivatives.

Then, we consider a $I P_{k}$ finite element discretization and we prove the existence, uniqueness and the convergence of the discrete solution.

Finally, we present some numerical results illustrating the mathematical properties proved and the solution behavior.

\section{The $1 \mathrm{D}$ steady-state LSM}

Let us consider the LSM (22), the model (19) being similar.

We assume that the boundary $\Gamma_{S L}^{m}$ is straight and parallel to a $x_{i}$-axis, $\mathrm{i}=1,2$.

We set $f=\frac{1}{\tau^{*}} \rho_{2}^{e q}, \rho^{*}=\rho_{2}^{e q}$ the characteristic surface density, $U^{*}=$ $\left|U_{S}\right|$ the characteristic velocity and let $l$ be the characteristic length of the mesoscopic length $\left|\Gamma_{S L}^{m}\right|$.

We set the dimensionless variable $\tilde{\rho}=\frac{\rho}{\rho^{*}}$ and we immediately omit the on the dimensionless variables.

The $1 \mathrm{D}$ steady-state and dimensionless LSM is the following:

$$
\left\{\begin{aligned}
-\left(\rho \rho^{\prime}\right)^{\prime}+\delta_{1} U \rho^{\prime}+\delta_{2} \rho & =\delta_{2} \quad \text { for } y \in(0,1) \\
\rho(0) & =1 \\
\left(-\rho \rho^{\prime}+\delta_{1} U \rho\right)(1) & =\phi
\end{aligned}\right.
$$

where

$$
\delta_{1}=\frac{l U^{*}}{\lambda \rho^{*}} \text { and } \delta_{2}=\frac{l^{2}}{\lambda \rho^{*} \tau^{*}}
$$


are dimensionless numbers,

$$
\phi=\delta_{1} \rho_{1}^{e q}(2 U(1)+1)
$$

is the flux at the contact point, and $U$ is defined by (20).

Let us notice that if we set $l=\tau^{*} U^{*}$, then $\delta_{1}=\delta_{2}=\frac{\tau^{*}\left(U^{*}\right)^{2}}{\lambda \rho^{*}}$.

\subsection{Mathematical analysis}

We consider the mathematical model:

$$
(P)\left\{\begin{array}{cl}
-\left(\rho \rho^{\prime}\right)^{\prime}+\delta_{1} U \rho^{\prime}+\delta_{2} \rho & =f \text { in }] 0,1[ \\
\rho(0) & =\rho_{0} \\
\left(-\rho \rho^{\prime}+\delta_{1} U \rho\right)(1) & =\phi
\end{array}\right.
$$

where $\delta_{i}>0, i=1,2$, and $f$ is given in $L^{2}(0,1)$.

Under some physical assumptions, we prove the existence of the weak solution of $(P)$ using the Leray-Schauder fixed point theorem, the uniqueness using the Gronwall's Lemma and we prove some extra properties of the first and second derivatives of the solution.

We make the following assumption on the velocity $U$.

\section{Assumption 3.1}

i) $U \in W^{1, \infty}(0,1)$ and $U \leq 0$ in $[0,1]$.

ii) $U^{\prime} \geq 0$ a.e. and $\left\|U^{\prime}\right\|_{\infty} \leq \frac{2 \delta_{2}}{\delta_{1}}$.

We will prove the existence and uniqueness of solution of $(P)$ using the Leray-Schauder fixed point theorem. To this end, we first prove the existence, uniqueness and some extra regularity of the solution of the following linearized and regularized problem.

Let $\varepsilon>0$, let $\beta_{\varepsilon} \in C^{1}(\mathbb{R})$, Lipschitz, increasing and such that:

$$
\beta_{\varepsilon}(x)=\left\{\begin{array}{lll}
\varepsilon & \text { if } & x \leq 0 \\
x & \text { if } & x \geq 2 \varepsilon
\end{array}\right.
$$

Let $\theta:[0,1] \rightarrow \mathbb{R}$ be given, the linearized and regularized problem is:

$$
\left(Q^{\beta}\right)\left\{\begin{array}{cl}
-\left(\beta_{\varepsilon}(\theta) \psi^{\prime}\right)^{\prime}+\delta_{1} U \psi^{\prime}+\delta_{2} \psi & =f \text { in }] 0,1[ \\
\psi(0) & =\rho_{0} \\
\left(-\beta_{\varepsilon}(\theta) \psi^{\prime}+\delta_{1} U \psi\right)(1) & =\phi
\end{array}\right.
$$


We set:

$$
\begin{gathered}
V_{0}=\left\{v \in H^{1}(0,1), v(0)=0\right\} \\
V_{\rho_{0}}=\left\{v \in H^{1}(0,1), v(0)=\rho_{0}\right\} \\
a_{2}^{\beta}(\theta ; \psi, v)=\int_{0}^{1} \beta_{\varepsilon}(\theta) \psi^{\prime} v^{\prime} d x \\
a_{1}(\psi, v)=\delta_{1}\left[\int_{0}^{1} U \psi^{\prime} v d x-U(1) \psi(1) v(1)\right] \\
a_{0}(\psi, v)=\delta_{2} \int_{0}^{1} \psi v d x \\
a^{\beta}(\theta ; \psi, v)=a_{2}^{\beta}(\theta ; \psi, v)+a_{1}(\psi, v)+a_{0}(\psi, v)
\end{gathered}
$$

and

$$
l(v)=\int_{0}^{1} f v d x-\phi v(1)
$$

With the notations above, the weak formulation is:

$$
\left(\mathcal{Q}^{\beta}\right)\left\{\begin{array}{l}
\text { Find } \psi \in V_{\rho_{0}} \text { such that } \\
a^{\beta}(\theta ; \psi, v)=l(v) \quad \forall v \in V_{0}
\end{array}\right.
$$

Proposition 3.1 Let $\theta \in H^{1}(0,1)$ be given and let Assumption 3.1 be satisfied.

i) There exists an unique solution $\psi$ to $\left(\mathcal{Q}^{\beta}\right)$.

ii) $\psi \in H^{2}(0,1)$.

Proof. i) We have $\theta \in H^{1}(0,1), \beta_{\varepsilon}(\theta)(x) \in\left[\varepsilon, \beta_{\varepsilon}\left(\|\theta\|_{\infty}\right)\right]$ and $U \in$ $W^{1, \infty}(0,1)$, then the continuity of $a^{\beta}(.,$.$) and l($.$) are straightforward.$

The bilinear form $a^{\beta}(\theta ; .,$.$) is V_{0}$-coercive: $\forall v \in V_{0}$,

$$
\begin{aligned}
a^{\beta}(\theta ; v, v)= & \int_{0}^{1} \beta_{\varepsilon}(\theta)\left(v^{\prime}\right)^{2} d x+\frac{\delta_{1}}{2}\left[\int_{0}^{1} U\left(v^{2}\right)^{\prime} d x-2 U(1) v^{2}(1)\right] \\
& +\delta_{2} \int_{0}^{1} v^{2} d x \\
= & \int_{0}^{1} \beta_{\varepsilon}(\theta)\left(v^{\prime}\right)^{2} d x-\delta_{1} U(1) v^{2}(1)+\int_{0}^{1}\left[\delta_{2}-\frac{\delta_{1}}{2} U^{\prime}\right] v^{2} d x
\end{aligned}
$$

Under Assumption 3.1,

$$
a^{\beta}(\theta ; v, v) \geq c_{\varepsilon}\|v\|_{1}^{2} \quad \forall v \in V_{0}
$$


where $c_{\varepsilon}$ is a constant independent of $\theta$.

Then, the result follows from the Lax-Milgram theorem.

ii) We have:

$$
-\left(\beta_{\varepsilon}(\theta) \psi^{\prime}\right)^{\prime}+\delta_{1} U \psi^{\prime}+\delta_{2} \psi=f \text { in } \mathcal{D}^{\prime}(0,1)
$$

with $U \in W^{1, \infty}(0,1)$ and $\beta_{\varepsilon}(\theta) \psi^{\prime} \in L^{\infty}(0,1)$. Hence $\beta_{\varepsilon}(\theta) \psi^{\prime} \in H^{1}(0,1)$ and one can deduce that $\psi^{\prime} \in H^{1}(0,1)$. Hence the result.

Now, we consider the non linear regularized problem:

$$
\left(P^{\beta}\right)\left\{\begin{array}{cl}
\text { Find } \rho \text { satisfying } & \\
-\left(\beta_{\varepsilon}(\rho) \rho^{\prime}\right)^{\prime}+\delta_{1} U \rho^{\prime}+\delta_{2} \rho & =f \text { in }] 0,1[ \\
\rho(0) & =\rho_{0} \\
\left(-\beta_{\varepsilon}(\rho) \rho^{\prime}+\delta_{1} U \rho\right)(1) & =\phi
\end{array}\right.
$$

Proposition 3.2 Under Assumption 3.1, Problem $\left(P^{\beta}\right)$ has at least one weak solution in $H^{1}(0,1)$ and this solution belongs to $H^{2}(0,1)$.

Proof. We use the Leray-Schauder fixed point theorem. To this end, we define the operator $T$ as follows:

$$
T: H^{1}(0,1) \rightarrow H^{1}(0,1) ; \theta \mapsto \psi
$$

where $\psi$ is the unique solution of $\left(\mathcal{Q}^{\beta}\right)$.

Any fixed point of $T$ is solution of $\left(P^{\beta}\right)$.

Using the continuity and the coercivity of the bilinear form $a^{\beta}(.,$.$) -see$ (32)-, we obtain: $\forall \theta \in H^{1}(0,1)$,

$$
\|\psi\|_{1}=\|T(\theta)\|_{1} \leq c_{\varepsilon}
$$

where $c_{\varepsilon}$ is a constant independent of $\theta$.

In others respects, as $\psi=T(\theta) \in H^{2}(0,1)$ (Proposition 3.1), we obtain from the first equation of $\left(Q^{\beta}\right)$ :

$$
-\beta_{\varepsilon}(\theta) \psi^{\prime \prime}-\beta_{\varepsilon}^{\prime}(\theta) \psi^{\prime}+\delta_{1} U \psi^{\prime}+\delta_{2} \psi=f
$$

Let us suppose that $\theta$ belongs to a ball $B(0, r)$ in $H^{1}(0,1)$. Then we deduce that

$$
\varepsilon\left\|\psi^{\prime \prime}\right\|_{2} \leq\left(r+\delta_{1}\|U\|_{\infty}\right)\left\|\psi^{\prime}\right\|_{0}+\delta_{2}\|\psi\|_{0}+\|f\|_{0}
$$

So $T(B(0, r))$ is bounded in $H^{2}(0,1)$. It follows from the compactness of the injection from $H^{2}(0,1)$ into $H^{1}(0,1)$, that $T$ is compact from $H^{1}(0,1)$ 
into itself.

Let $\sigma$ in $[0,1]$ be given and $\rho$ be a fixed point of $\sigma T: \rho=\sigma T(\rho)$.

We set $\tilde{\rho}=\left(\rho-\rho_{0}\right), \tilde{\rho} \in V_{0}$. As previously, we prove that there exists a constant $c_{\varepsilon}$ independent of $\sigma$ such that:

$$
\|\tilde{\rho}\|_{1} \leq c_{\varepsilon}
$$

It follows from the Leray-Schauder theorem (see e.g. [7]) that $T$ has at least one fixed point $\rho$ in $H^{1}(0,1)$ and the result follows.

Let us assume

Assumption 3.2 We have: $\rho_{0}>0, \phi \leq 0$ and $\inf _{(0,1)} f>0$.

Let $\eta$ be a real satisfying: $\rho_{0} \geq \eta>0, \eta \delta_{2} \leq \inf _{(0,1)}(f)$ and $\eta \delta_{1} U(1) \geq$ $\phi$.

For example for $U(1)<0$, we set:

$$
\eta=\min \left\{\rho_{0}, \frac{1}{\delta_{2}} \inf _{(0,1)}(f), \frac{\phi}{\delta_{1} U(1)}\right\}
$$

Lemma 3.1 Under assumptions 3.1 and 3.2 , the unique solution $\psi$ of $\left(\mathcal{Q}^{\beta}\right)$ satisfies $\psi \geq \eta>0$ in $[0,1]$.

Proof. We choose $v=(\psi-\eta)^{-}=\max (-\psi+\eta, 0)$ as test function. We have $v$ in $V_{0}, v \geq 0, v=(-\psi+\eta)$ if $\eta \geq \psi$ and $v=0$ if $\eta<\psi$.

Under Assumption 3.2 and using the equality $\psi v=(\eta-v) v$, we obtain:

$$
\begin{array}{r}
-\int_{0}^{1} \beta_{\varepsilon}(\theta)\left(v^{\prime}\right)^{2} d x+\frac{\delta_{1}}{2} U(1) v^{2}(1)-\int_{0}^{1}\left[\delta_{2}-\frac{\delta_{1}}{2} U^{\prime}\right] v^{2} d x \\
=\int_{0}^{1}\left[f-\eta \delta_{2}\right] v d x+\left[\eta \delta_{1} U(1)-\phi\right] v(1) \\
\geq 0
\end{array}
$$

Under Assumption 3.1, we have $U(1) \leq 0$ and $\left(\delta_{2}-\frac{\delta_{1}}{2} U^{\prime}\right) \geq 0$, hence

$$
\int_{0}^{1} \beta_{\varepsilon}(\theta)\left(v^{\prime}\right)^{2} d x=0
$$

It follows that $v=(\psi-\eta)^{-}=0$ and $\psi \geq \eta$. 
Theorem 3.1 Under assumptions 3.1 and 3.2, Problem $(P)$ has at least one weak solution $\rho$ in $H^{1}(0,1)$. This solution satisfies $\rho(x) \geq \eta>0$ in $[0,1]$ and belongs to $H^{2}(0,1)$.

Proof. It is straightforward. Let us choose $\varepsilon=\frac{\eta}{2}$-see (26) and (34)-. Let $T$ be the operator defined by (33), for all $\theta \in H^{1}(0,1), \psi=T(\theta) \geq \eta>0$ -Lemma 3.1-. Also, $\rho=T(\rho) \geq 2 \varepsilon$ and $\beta_{\varepsilon}(\rho)=\rho$. Hence the result.

Theorem 3.2 Under assumptions 3.1 and 3.2 , and if $\left\|U^{\prime}\right\|_{\infty} \leq \frac{\delta_{2}}{\delta_{1}}$, Problem $(P)$ has an unique solution $\rho$.

Proof. We denote by $\rho_{1}$ and $\rho_{2}$ two solutions of $(P)$.

a) First, we prove that $\rho_{1}^{\prime}(0)=\rho_{2}^{\prime}(0)$.

Let us suppose that $\rho_{1}^{\prime}(0)>\rho_{2}^{\prime}(0)$. Let $] 0, \xi_{0}$ ] the largest interval such that $\left.\rho_{1}(x)>\rho_{2}(x), x \in\right] 0, \xi_{0}[$.

Let us suppose $\xi_{0}=1$. We integrate the first equation of $(P)$ on $[0,1]$ with $\rho_{1}$ and $\rho_{2}$. By differentiating we obtain:

$$
\begin{array}{r}
-\rho_{1} \rho_{1}^{\prime}(1)+\rho_{2} \rho_{2}^{\prime}(1)+\rho_{0}\left(\rho_{1}^{\prime}(0)-\rho_{2}^{\prime}(0)\right)+\delta_{1}\left[U\left(\rho_{1}-\rho_{2}\right)\right]_{0}^{1} \\
+\int_{0}^{1}\left(\delta_{2}-\delta_{1} U^{\prime}\right)\left(\rho_{1}-\rho_{2}\right) d x=0
\end{array}
$$

Using the boundary conditions of $(P)$, we obtain:

$$
\rho_{0}\left(\rho_{1}^{\prime}(0)-\rho_{2}^{\prime}(0)\right)+\int_{0}^{1}\left(\delta_{2}-\delta_{1} U^{\prime}\right)\left(\rho_{1}-\rho_{2}\right) d x=0
$$

hence

$$
\int_{0}^{1}\left(\delta_{2}-\delta_{1} U^{\prime}\right)\left(\rho_{1}-\rho_{2}\right) d x<0
$$

which is impossible.

Therefore $\left.\xi_{0} \in\right] 0,1\left[\right.$ and $\rho_{1}\left(\xi_{0}\right)=\rho_{2}\left(\xi_{0}\right)$. As previously, we integrate on $\left[0, \xi_{0}\right]$ and we obtain:

$$
\begin{array}{r}
-\rho_{1}\left(\xi_{0}\right)\left(\rho_{1}^{\prime}\left(\xi_{0}\right)-\rho_{2}^{\prime}\left(\xi_{0}\right)\right)+\rho_{0}\left(\rho_{1}^{\prime}(0)-\rho_{2}^{\prime}(0)\right) \\
+\int_{0}^{1}\left(\delta_{2}-\delta_{1} U^{\prime}\right)\left(\rho_{1}-\rho_{2}\right) d x=0
\end{array}
$$

hence $\left(\rho_{1}-\rho_{2}\right)^{\prime}\left(\xi_{0}\right)>0$. It is impossible. Therefore, $\rho_{1}^{\prime}(0)=\rho_{2}^{\prime}(0)$.

b) Second, we write the first equation of $(P)$ as a first order differential equation of the form:

$$
\left.W^{\prime}(x)=G(W)(x), x \in\right] 0,1[
$$


with $W=(u, v)^{T}$ and

$$
G(W)(s)=\left(v(s), \frac{-1}{u(s)}\left[-v^{2}(s)+\delta_{1} U(s) v(s)+\delta_{2} u(s)-f(s)\right]\right)^{T}
$$

We consider $G: C^{1}([0,1] ; \mathbb{R}) \cap \mathcal{F}^{+} \times C^{0}([0,1] ; \mathbb{R}) \rightarrow C^{0}([0,1] ; \mathbb{R}) \times$ $C^{0}([0,1] ; \mathbb{R})$ with $\mathcal{F}^{+}=\left\{u, u \in C^{0}([0,1] ; \mathbb{R}), u>0\right.$ in $\left.[0,1]\right\}$.

One can easily verify that $G(W)$ is of class $C^{1}$.

Then, we set $W_{i}=\left(\rho_{i}, \rho_{i}^{\prime}\right)^{T}, i=1,2$. We have $W_{i}^{\prime}(x)=G\left(W_{i}\right)(x), x \in$ ]0, $1\left[\right.$, and $W_{1}(0)=W_{2}(0)$. Hence,

$$
\left(W_{1}-W_{2}\right)(x)=\int_{0}^{x}\left(G\left(W_{1}\right)-G\left(W_{2}\right)\right)(s) d s
$$

Since $\rho_{i} \in C^{1}([0,1] ; \mathbb{R}) \cap \mathcal{F}^{+}$and $G\left(W_{i}\right)$ is of class $C^{1}$, there exists a constant $k$ such that:

$$
\left\|\left(G\left(W_{1}\right)-G\left(W_{2}\right)\right)(s)\right\| \leq k\left\|\left(W_{1}-W_{2}\right)(s)\right\|
$$

Then, it follows from (35) that:

$$
\left\|\left(W_{1}-W_{2}\right)(x)\right\| \leq k \int_{0}^{x}\left\|\left(W_{1}-W_{2}\right)(s)\right\| d s
$$

Setting $I(x)=\int_{0}^{x}\left\|\left(W_{1}-W_{2}\right)(s)\right\| d s$, we obtain: $\frac{d}{d x}(I(x) \exp (-k x)) \leq 0$. Also, $I(0)=0$, therefore $I(x) \leq 0 \forall x \in[0,1]$.

Then, we conclude that $W_{1}=W_{2}$ in $[0,1]$.

Now, we make

Assumption 3.3 We have $f=\delta_{2} \rho_{0}$ and $(U, \phi)$ such that: $U(0)>2 U(1)$, $\left\|U^{\prime}\right\|_{\infty} \leq \frac{\delta_{2}}{\delta_{1}}$ and $\phi=\delta_{1} \tilde{\rho}(2 U(1)-U(0)) \leq 0$ with $\tilde{\rho}>0$.

Under Assumption 3.3, $\eta$ defined by (34) becomes:

$$
\eta=\min \left\{\rho_{0}, \frac{\phi}{\delta_{1} U(1)}\right\}
$$

Under these assumptions, we prove that the solution is monotone and we prove some extra properties on $\rho^{\prime}$ and $\rho^{\prime \prime}$.

Theses properties are useful for the numerical analysis presented in next section too. 
Proposition 3.3 Let the assumptions $3.1,3.2$ and 3.3 be satisfied and let $\rho$ be the unique solution of $(P)$.

i) If $\phi=\delta_{1} U(1) \rho_{0}$ then $\rho \equiv \rho_{0}$.

ii) If $\phi<\delta_{1} U(1) \rho_{0}$ then $\rho(x) \geq \rho_{0}$ and $\rho^{\prime}(x) \geq 0$ in $[0,1]$.

iii) If $\phi>\delta_{1} U(1) \rho_{0}$ then $\rho(x) \leq \rho_{0}$ and $\rho^{\prime}(x) \leq 0$ in $[0,1]$

Proof.

i) It is straightforward to verify that $\rho \equiv \rho_{0}$ is solution and the solution is unique.

ii) We have $\phi<\delta_{1} U(1) \rho_{0}$. Since $\psi \geq \eta$ in $[0,1]$ (Lemma 3.1), we obtain $\rho \geq \rho_{0}$ in $[0,1]$. Let us prove that $\rho^{\prime}(x) \geq 0$.

If there exists $\left.\xi_{0} \in\right] 0,1\left[\right.$ such that $\rho^{\prime}\left(\xi_{0}\right)<0$, then

$$
-\left(\rho \rho^{\prime}\right)^{\prime}\left(\xi_{0}\right)+\delta_{1} U\left(\xi_{0}\right) \rho^{\prime}\left(\xi_{0}\right)=\delta_{2}\left(\rho_{0}-\rho\left(\xi_{0}\right)\right) \leq 0
$$

hence

$$
-\left(\rho \rho^{\prime}\right)^{\prime}\left(\xi_{0}\right)=-\frac{1}{2}\left(\rho^{2}\right)^{\prime}\left(\xi_{0}\right)<0
$$

We deduce that $\left(\rho^{2}\right)^{\prime}(\xi)>0$ in a neighborhood $\mathcal{V}\left(\xi_{0}\right)$.

Therefore $\rho$ is increasing in $\mathcal{V}\left(\xi_{0}\right)$ and $\rho^{\prime}(\xi) \geq 0$ in $\mathcal{V}\left(\xi_{0}\right)$, which is a contradiction with $\rho^{\prime}\left(\xi_{0}\right)<0$.

Then, we deduce that $\rho^{\prime}(x) \geq 0$ in $] 0,1[$ hence in $[0,1]$ since it is continue.

iii) We have $\phi>\delta_{1} U(1) \rho_{0}$. Let us prove that $\rho \leq \rho_{0}$ in $[0,1]$. To this end, we suppose that $\max _{[0,1]} \rho(\xi)=\rho\left(\xi_{m}\right)>\rho_{0}$.

We have $\xi_{m} \neq 0$ since $\rho(0)=\rho_{0}$. Let us suppose $\left.\xi_{m} \in\right] 0,1[$.

We write the equation in $\xi_{m}$ :

$$
-\left(\rho \rho^{\prime}\right)^{\prime}\left(\xi_{m}\right)+\delta_{1} U\left(\xi_{m}\right) \rho^{\prime}\left(\xi_{m}\right)+\delta_{2} \rho\left(\xi_{m}\right)=\delta_{2} \rho_{0}
$$

hence

$$
-\rho \rho^{\prime \prime}\left(\xi_{m}\right)=\delta_{2}\left(\rho_{0}-\rho\left(\xi_{m}\right)\right)
$$

since $\rho^{\prime}\left(\xi_{m}\right)=0$. We deduce $-\rho \rho^{\prime \prime}\left(\xi_{m}\right)<0$ and $\rho^{\prime \prime}\left(\xi_{m}\right)>0$. It is a contradiction with the definition of $\xi_{m}$.

Let us suppose $\xi_{m}=1$. We have $\rho^{\prime}\left(\xi_{m}\right) \geq 0$. Using the boundary condition in $x=1$, we obtain: $\phi \leq \delta_{1} U(1) \rho(1)$. In others respects, $\phi>\delta_{1} U(1) \rho_{0}>\delta_{1} U(1) \rho(1)$.

It leads to a contradiction. Therefore, $\rho \leq \rho_{0}$ in $[0,1]$.

The proof of $\rho^{\prime} \leq 0$ in $[0,1]$ is similar to the previous case ii). 
Lemma 3.2 Let the assumptions 3.1, 3.2 and 3.3 be satisfied and let $\rho$ be the unique solution of $(P)$. We have: $\left(\rho^{\prime}-\delta_{1} U\right)(x) \geq 0$ in $[0,1]$.

Proof. Since $U<0$ in $[0,1]$, the inequality is obvious if $\rho^{\prime} \geq 0$ in $[0,1]$. Let $\rho^{\prime} \leq 0$ in $[0,1]$. We have:

$$
\left(-\rho \rho^{\prime}+\delta_{1} U \rho\right)^{\prime}-\delta_{1} U^{\prime} \rho=\delta_{2}\left(\rho_{0}-\rho\right) \geq 0
$$

and the function $\tau \mapsto\left(-\rho \rho^{\prime}+\delta_{1} U \rho\right)(\tau)$ is increasing. Therefore,

$$
\forall x \in[0,1], \quad-\rho(x)\left[\rho^{\prime}(x)-\delta_{1} U(x)\right] \leq \phi \leq 0
$$

hence the result.

Lemma 3.3 Let the assumptions $3.1,3.2$ and 3.3 be satisfied and let $\rho$ be the unique solution of $(P)$.

i) If $\phi \leq \delta_{1} U(1) \rho_{0}$ then $\rho^{\prime \prime}(x) \leq \delta_{2}$ in $[0,1]$.

ii) If $\phi>\delta_{1} U(1) \rho_{0}$ and $U^{2}(0) \leq 4 \frac{\delta_{2}}{\delta_{1}^{2}} \rho_{0}$ then $\rho^{\prime \prime}(x) \leq \delta_{2}$ in $[0,1]$.

Proof.

i) We have $\rho(x) \geq \rho_{0}$ and $\rho^{\prime}(x) \geq 0 \quad \forall x \in[0,1]$ (Lemma 3.2). We deduce from the first equation of $(P)$ :

$$
\rho(x)\left[\delta_{2}-\rho^{\prime \prime}(x)\right]=\rho^{\prime 2}(x)-\delta_{1} U(x) \rho^{\prime}(x)+\delta_{2} \rho_{0}
$$

The right hand side is positive and the result follows.

ii) We have $\rho^{\prime}(x) \leq 0$ and $\rho(x)>0$ in $[0,1]$.

We have to control the sign of the right hand side of (39). The trinom $X^{2}-\delta_{1} U(x) X+\delta_{2} \rho_{0}$ is positive for all $x$ in $[0,1]$ if and only if $\Delta(x)=\left(\delta_{1} U(x)\right)^{2}-4 \delta_{2} \rho_{0} \leq 0$.

Therefore under Assumption 3.1 and if $\delta_{1} U(0)^{2} \leq 4 \delta_{2} \rho_{0}$, the result follows.

Proposition 3.4 Let the assumptions $3.1,3.2$ and 3.3 be satisfied. Let $A$ be the operator defined by: $A: \mathbb{R} \rightarrow H^{1}(0,1) ; \phi \mapsto \rho$, where $\rho$ is the unique solution of $(P)$. Then, the operator $A$ is monotone.

Namely, let $\phi_{2}<\phi_{1}<0$, let $\rho_{1}$ and $\rho_{2}$ be the unique corresponding solutions, then we have: $\rho_{2} \geq \rho_{1}$ in $[0,1]$.

Proof.

Case 1. We have: $\phi_{2}<\delta_{1} U(1) \rho_{0} \leq \phi_{1}<0$

In vertu of Proposition 3.3, $\rho_{1} \leq \rho_{0} \leq \rho_{2}$ and the property holds. 
Case 2. We have: $\delta_{1} U(1) \rho_{0}<\phi_{2}<\phi_{1}$.

A. Let us prove that $\rho_{1}^{\prime}(0) \leq \rho_{2}^{\prime}(0)$.

In vertu of Proposition 3.3, we have $\rho_{i}(x) \leq \rho_{0}$ and $\rho_{i}^{\prime}(x) \leq 0, i=1,2$, in $[0,1]$.

Let us suppose that $\rho_{1}^{\prime}(0)>\rho_{2}^{\prime}(0)$. Then, there exists $\xi$ such that $\rho_{1}>\rho_{2}$ in $] 0, \xi$. Let $\xi_{\text {max }}$ be the largest value of $\xi$ such that $\rho_{1}>\rho_{2}$ in ] $0, \xi[$.

a) Case $\xi_{\text {max }}=1$.

Following the same idea of proof than in Theorem 3.2, we integrate the first equation of $(P)$ on $[0,1]$ with $\rho_{1}$ and $\rho_{2}$, we differentiate the two equations and we obtain:

$$
\left(\phi_{1}-\phi_{2}\right)+\rho_{0}\left(\rho_{1}^{\prime}(0)-\rho_{2}^{\prime}(0)\right)+\int_{0}^{1}\left(\delta_{2}-\delta_{1} U^{\prime}\right)\left(\rho_{1}-\rho_{2}\right) d x=0
$$

which leads to a contradiction. Hence the case $\xi_{\max }=1$ is impossible.

b) Case $\left.\xi_{\max } \in\right] 0,1[$.

We have: $\rho_{1}\left(\xi_{\text {max }}\right)=\rho_{2}\left(\xi_{\text {max }}\right)=\lambda>0$.

We integrate the first equation of $(P)$ in $] 0, \xi_{\max }[$ and we proceed as previously. We obtain:

$-\lambda\left[\left(\rho_{1}^{\prime}-\rho_{2}^{\prime}\right)\left(\xi_{\text {max }}\right)\right]+\rho_{0}\left(\rho_{1}^{\prime}-\rho_{2}^{\prime}\right)(0)+\int_{0}^{\xi_{\max }}\left(\delta_{2}-\delta_{1} U^{\prime}\right)\left(\rho_{1}-\rho_{2}\right) d x=0$

Then, we deduce: $\left(\rho_{1}^{\prime}-\rho_{2}^{\prime}\right)\left(\xi_{\max }\right)>0$. Hence, $\left(\rho_{1}-\rho_{2}\right)$ is increasing in a neighborhood of $\xi_{\text {max }}$, which is in contradiction with $\left(\rho_{1}-\rho_{2}\right)\left(\xi_{\text {max }}\right)=0$ and $\left(\rho_{1}-\rho_{2}\right)(x)>0$ in $] 0, \xi_{\max }[$.

It follows that: $\rho_{1}^{\prime}(0) \leq \rho_{2}^{\prime}(0)$.

B. Following the proof of Theorem 3.2, we have: $\rho_{1}^{\prime}(0)=\rho_{2}^{\prime}(0)$ implies $\rho_{1}=\rho_{2}$ in $[0,1]$. Since $\phi_{1}$ not equal to $\phi_{2}$, necessarily, $\rho_{1}^{\prime}(0)<\rho_{2}^{\prime}(0)$.

Hence, there exists $\tau$ such that $\rho_{2}>\rho_{1}$ in $] 0, \tau\left[\right.$. Let $\tau_{\max }$ be the largest value of $\tau$ such that $\rho_{2}>\rho_{1}$ in $] 0, \tau[$.

Let us suppose $\tau_{\max }<1$. Then, $\rho_{1}\left(\tau_{\max }\right)=\rho_{2}\left(\tau_{\max }\right)$ and $\rho_{1}^{\prime}\left(\tau_{\max }\right) \geq$ $\rho_{2}^{\prime}\left(\tau_{\max }\right)$.

Let us consider the case $\rho_{1}^{\prime}\left(\tau_{\max }\right)=\rho_{2}^{\prime}\left(\tau_{\max }\right)$. Theorem 3.2 as applied to the interval $\left[\tau_{\max }, 1\right]$ gives $\rho_{1}=\rho_{2}$ and $\phi_{1}=\phi_{2}$; which is impossible. Therefore, $\rho_{1}^{\prime}\left(\tau_{\max }\right)>\rho_{2}^{\prime}\left(\tau_{\max }\right)$. We follow the same idea of proof than 
previously. There exists an interval $] \tau_{\max }, \xi\left[\right.$ such that $\rho_{1}>\rho_{2}$. Let ]$\tau_{\max }, \xi_{\max }[$ be the largest interval such that the property holds. We consider separately the two cases $\xi_{\max }=1$ and $\xi_{\max }<1$ and by integrating the first equation of Problem $(P)$, we conclude to a contradiction. Finally, we obtain that $\tau_{\max }=1$ and $\rho_{2} \geq \rho_{1}$ in $\left.] 0,1\right]$.

Case 3. For this case, we have: $\phi_{2}<\phi_{1}<\delta_{1} U(1) \rho_{0}$.

The proof is similar to case 2 . Hence, the result.

\subsection{Finite element numerical analysis}

We discretize the 1D steady-state LSM using the Lagrange finite element method. The numerical analysis is done using the framework and results for non-linear problems presented in [2]. We obtain a local existence, uniqueness and convergence of the finite element solution.

Setting $\rho=\left(\theta+\rho_{0}\right)$, the weak formulation of Problem $(P)$ is written as follows:

$$
(\mathcal{P})\left\{\begin{array}{l}
\text { Find } \theta \in V_{0} \text { such that: } \\
b(\theta ; \theta, v)=m(v) \quad \forall v \in V_{0}
\end{array}\right.
$$

where $V_{0}$ is defined by $(27)$,

$$
b(\theta ; \psi, v)=a_{2}\left(\theta+\rho_{0} ; \psi, v\right)+a_{1}(\psi, v)+a_{0}(\psi, v)
$$

with

$$
a_{2}(\rho ; \psi, v)=\int_{0}^{1} \rho \psi^{\prime} v^{\prime} d x
$$

with $a_{1}(.,$.$) and a_{0}(.,$.$) defined by (29)$ and (30) respectively,

$$
m(v)=l(v)-\delta_{2} \rho_{0} \int_{0}^{1} v d x
$$

and $l($.$) defined by (31).$

Let us recall that under assumptions $3.1,3.2$ and 3.3 , there exists an unique solution to $(\mathcal{P})$. Furthermore, this solution belongs to $H^{2}(0,1)$ and satisfies: $\rho(x)=\theta(x)+\rho_{0} \geq \eta>0$ in $[0,1]$ (Theorems 3.1 and 3.2 ).

We define the operator

$$
F: V_{0} \rightarrow V_{0}^{\prime} \text { by: }\left\langle F(\theta), v>=b(\theta ; \theta, v)-m(v) \forall v \in V_{0}\right.
$$


where $\langle.,$.$\rangle denotes the duality product V_{0}^{\prime} \times V_{0}$.

Problem $(\mathcal{P})$ is equivalent to:

$$
\left\{\begin{array}{l}
\text { Find } \theta \in V_{0} \text { such that: } \\
\langle F(\theta), v\rangle=0 \quad \forall v \in V_{0}
\end{array}\right.
$$

We discretize Problem $(\mathcal{P})$ using a $P_{k}$ Lagrange finite element method $(k \in \mathbb{N}, k \geq 1)$.

To this end, we set: $[0,1]=U_{(i=0 . .(N-1))}\left[x_{i}, x_{i+1}\right]$ and $h=\min _{(i)}\left(x_{i+1}-x_{i}\right)$. We define the finite element spaces:

$$
V_{0 h}=\left\{t \in \mathcal{C}^{0}([0,1]) ; \forall i,\left.t\right|_{\left[x_{i}, x_{i+1}\right]} \in P_{k} ; t(0)=0\right\},
$$

The discrete model is:

$$
\left(\mathcal{P}_{\rho h}\right)\left\{\begin{array}{l}
\text { Find } \theta_{h} \in V_{0 h} \text { such that: } \\
\left\langle F_{h}(\theta), v_{h}\right\rangle=0 \quad \forall v_{h} \in V_{0 h}
\end{array}\right.
$$

In order to apply the results for non-linear problems presented in [2], we first study the linearized problem.

The operator $F($.$) is of class C^{1}$ from $V_{0}$ into $V_{0}^{\prime}$.

Let $\theta$ be the unique solution of $(\mathcal{P})$, we study the following linearized problem:

$$
\left\{\begin{array}{l}
\text { Given } f \in V_{0}^{\prime} \text {, find } \kappa \in V_{0} \text { such that: } \\
\langle D F(\theta) . \kappa, v\rangle=\langle f, v\rangle \quad \forall v \in V_{0}
\end{array}\right.
$$

Lemma 3.4 Let the assumptions $3.1,3.2,3.3$ be satisfied and $U^{2}(0) \leq$ $4 \frac{\delta_{2}}{\delta_{1}^{2}} \rho_{0}$.

Let $\theta$ be the unique solution of $(\mathcal{P})$, the operator $D F(\theta)$ satisfies the following properties:

i) it is an isomorphism from $V_{0}$ into $V_{0}^{\prime}$,

ii) it is Lispchitzian at $\theta$, that is there exists $L>0$ such that for all $\kappa \in V_{0}$, $\|D F(\theta)-D F(\kappa)\|_{\mathcal{L}\left(V_{0} \times V_{0}^{\prime}\right)} \leq L\|\theta-\kappa\|_{1}$.

Proof

i) We claim that the bilinear form $\left\langle D F(\theta)\right.$., . $>$ is $V_{0}$-coercitive. We have:

$$
<D F(\theta) . \kappa, v>=b(\theta ; \kappa, v)+a_{2}(\kappa ; \theta, v)
$$

Using the same calculus as in Proposition 3.1, we obtain: $\forall v \in V_{0}$,

$$
\langle D F(\theta) \cdot v, v\rangle=\int_{0}^{1}\left(\theta+\rho_{0}\right)\left(v^{\prime}\right)^{2} d x+\int_{0}^{1} W_{\theta^{\prime \prime}} v^{2} d x+Z_{\theta^{\prime}} v^{2}(1)
$$


with: $W_{\theta^{\prime \prime}}(x)=\left(\delta_{2}-\frac{\delta_{1}}{2} U^{\prime}-\frac{1}{2} \theta^{\prime \prime}\right)(x)$ and $Z_{\theta^{\prime}}=\left(\frac{1}{2} \theta^{\prime}-\delta_{1} U\right)(1)$.

Under assumptions 3.1, 3.2, 3.3, since lemmas 3.2 and 3.3 hold, we have: $W_{\theta^{\prime \prime}} \geq 0$ and $Z_{\theta^{\prime}} \geq 0$. Therefore: $\forall v \in V_{0}$,

$$
\langle D F(\theta) \cdot v, v\rangle \geq c_{1}\left\|v^{\prime}\right\|_{0}^{2} \geq c_{2}\|v\|_{1}^{2}
$$

with $c_{i}>0, i=1,2$, independent of $v$. Then, the linearized problem is well posed in vertu of the Lax-Milgram theorem.

ii) The estimate is straightforward. We have: $\forall \kappa \in V_{0}, \forall(w, z) \in$ $V_{0} \times V_{0}$,

$$
\begin{aligned}
|<D F(\theta) \cdot w, z>-<D F(\kappa) \cdot w, z>| & =\left|a_{2}(\theta-\kappa ; w, z)+a_{2}(w ; \theta-\kappa, z)\right| \\
\leq & \leq\|\theta-\kappa\|_{1}\|w\|_{1}\|z\|_{1}
\end{aligned}
$$

and the property follows.

We have

Theorem 3.3 Let the assumptions $3.1,3.2,3.3$ be satisfied and $U^{2}(0) \leq$ $4 \frac{\delta_{2}}{\delta_{1}^{2}} \rho_{0}$.

There exists two constants $h_{0}>0, \alpha_{0}>0$, and for $h \leq h_{0}$, there exists an unique solution $\theta_{h}$ to problem $\left(\mathcal{P}_{\rho h}\right)$ in the closed ball $\bar{B}\left(\theta, \alpha_{0}\right)$.

Moreover, there exists a constant $c$ independent of $h$ such that:

$$
\left\|\theta-\theta_{h}\right\|_{1} \leq c \inf _{v_{h} \in V_{h}}\left\|\theta-v_{h}\right\|_{1} \leq c h\|\theta\|_{2}
$$

Proof. The result follows straightforwardly from the application of ([2], Theorem 7.1.). As a matter of fact, since Theorem 3.1, Theorem 3.2 and Lemma 3.4 hold, since the bilinear form $\langle D F(\theta) .,$.$\rangle is V_{0}$-coercitive, the estimates proved in ([2], Theorem 7.1.) hold with the constant $\beta_{h}=1$. Then, (40) holds since $\theta$ belongs to $H^{2}(0,1)$.

\subsection{Numerical results}

We compute the solution of the mesoscopic model $(P)$ using a finite difference method. We present two tests visualizing and confirming numerically the properties proved in the mathematical analysis section. The goal of these numerical results being a better understanding of the behavior of the solution $\rho$. As a matter of fact, in view to adjust the LSM parameters and to proceed to the full model fitting, one needs to perfectly understand the 
behavior of the LSM solution.

The numerical solution of $(P)$ is computed as the steady-state of the unsteady system. The time scheme is the Crank-Nicholson scheme and the non-linear term $\left(-\rho \rho^{\prime}\right)$ is linearized using the Newton-Raphson method. The code has been fully tested with explicit time-dependent solutions.

Let us summarize assumptions 3.1, 3.2 and 3.3. Data are such that:

- $U(x) \leq 0, U^{\prime}(x) \geq 0, U^{\prime}(x) \leq \frac{\delta_{2}}{\delta_{1}}$ in $[0,1]$ and $U(0)>2 U(1)$.

. $f=\delta_{2} \rho_{0}$ and $\phi=\delta_{1} \tilde{\rho}(2 U(1)-U(0)) \leq 0$,

with $\delta_{1}, \delta_{2}, \rho_{0}$ and $\tilde{\rho}$ strictly positives.

We set

$$
\eta=\min \left(\rho_{0}, \tilde{\rho}\left(2-\frac{U(0)}{U(1)}\right)\right)
$$

and we set

$$
\tilde{\rho}=\alpha \times \frac{\rho_{0}}{\left(2-\frac{U(0)}{U(1)}\right)}
$$

with $\alpha>0$.

The condition $\phi=\delta_{1} U(1) \rho_{0}$ (resp. $>$ and $<$ ) is equivalent to $\alpha=1$ (resp. $<$ and $>$ ), see Proposition 3.3.

Let us recall that for $\alpha=1$, the unique solution is $\rho(x) \equiv \rho_{0} \forall x$.

We have tested the two test cases a. and b. presented in Table 1, with $U(x)=\left(\frac{1}{2} x^{2}-1\right)$. All the assumptions are satisfied for both cases.

\begin{tabular}{|c|c|c|c|c|}
\hline Test No & $\delta_{1}$ & $\delta_{2}$ & $\rho_{0}$ & coef. $\alpha$ \\
\hline \hline a. & 5 & 1 & 1 & 10 \\
\hline b. & 1 & 10 & 1 & 0.1 \\
\hline
\end{tabular}

Table 1: Parameters for tests a. and b.

In Test a., we have $\alpha>1$ and in Test b., $\alpha<1$. The numerical results of Test a. (resp. Test b.) are presented in Fig. 3.3 (resp. Fig. 3.3).

In both cases, we obtained numerically the properties proved previously, namely: 

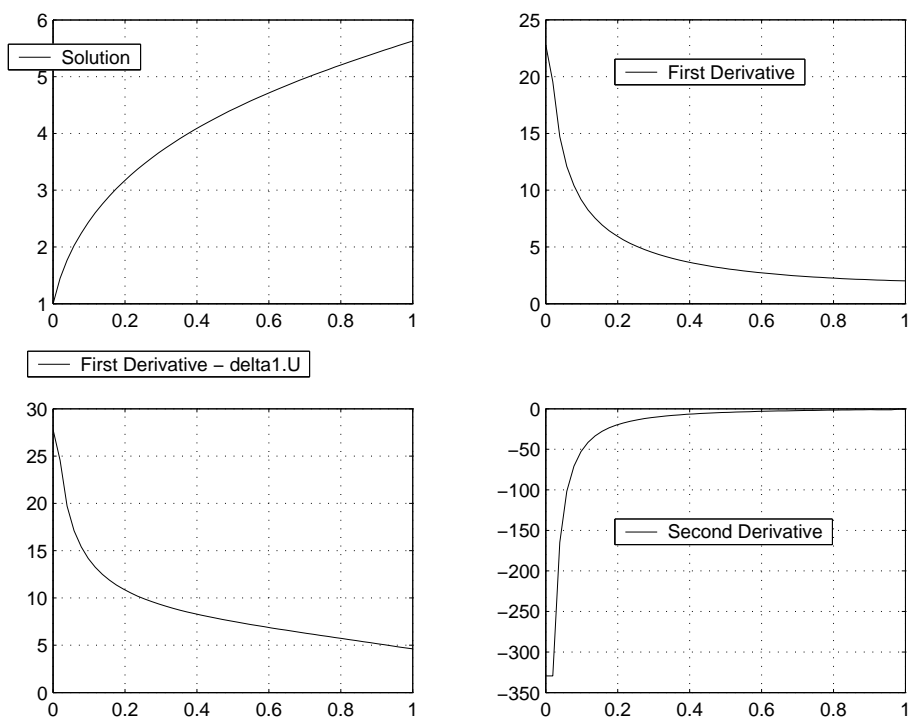

Figure 3: Test a.: $\rho, \rho^{\prime},\left(\rho^{\prime}-\delta_{1} U\right)$ and $\rho^{\prime \prime}$ plotted
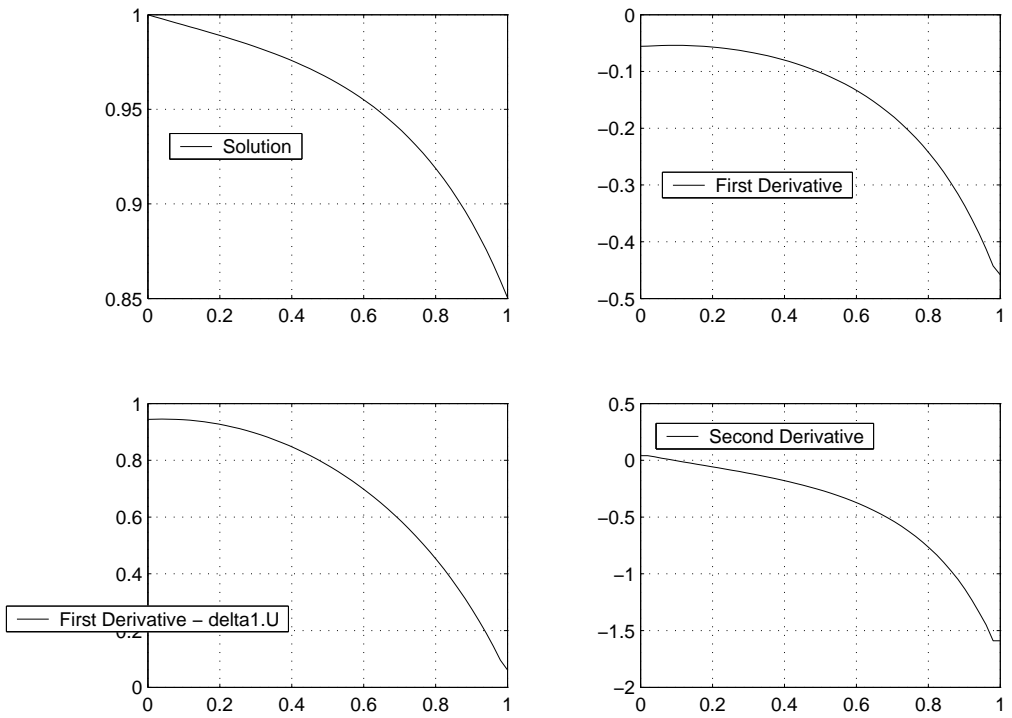

Figure 4: Test b.: $\rho, \rho^{\prime},\left(\rho^{\prime}-\delta_{1} U\right)$ and $\rho^{\prime \prime}$ plotted 
- $\rho(x) \geq \eta \forall x,(\eta=1$ for Test a. and 0.1 for Test b. $)$-Theorem 3.1-,

- $\phi-\delta_{1} U(1) \rho_{0}>0($ resp. $<0)$ implies $\rho(x) \geq \rho_{0}($ resp. $\leq 0)$ and $\rho^{\prime}(x) \geq 0 \forall x$ (resp. $\left.\leq 0\right)$-Proposition 3.3-,

- $\left(\rho^{\prime}-\delta_{1} U\right)(x) \geq 0 \quad \forall x$ - Lemma 3.2-

- $\left(\rho^{\prime \prime}(x)-\delta_{2}\right) \leq 0 \quad \forall x$-Lemma 3.3-.

The sign of $\rho^{\prime \prime}$ is not constant.

\section{Numerical Results: Plunging Tape Case Test}

We consider a 2D plunging tape configuration, and we solve numerically and separately the 1D mesoscopic LSM and the 2D macroscopic HFSM. In Section 4.1, the computation of the 1D mesoscopic LSM provides a profile of $\nabla \sigma_{2}$ : the local Marangoni source term in the Navier-Stokes boundary conditions.

In Section 4.2, we solve numerically a simplified version of the HFSM, namely a $2 \mathrm{D}$ Stokes model with the free surface $\Gamma_{L G}$ given and fixed. Then, we can observe the influence of $\nabla \sigma_{2}$ (the previous local Marangoni term) on the fluid motion in the bulk.

\section{The 2D plunging tape configuration}

The plunging tape case test is schematized in Figure 4. A tape plunges vertically into a pool of liquid at speed $U_{S}$. We consider an air-water-glass system.

We simplify the mesoscopic LSM as follows: we set $\sigma_{L G}=\sigma_{L G}^{e q}$. It follows from (15) that $v_{1}^{s}=u$ on $\Gamma_{L G}$. Then, the LSM is reduced to a 1D differential equation in an interval of the $\mathrm{y}$-axis $\left(\right.$ on $\left.\Gamma_{S L}^{m}\right)$.

\subsection{D LSM: local surface tension gradient}

The LSM considered is the following:

$$
\left\{\begin{array}{cll}
-\left(\rho \rho^{\prime}\right)^{\prime}+\delta_{1} U \rho^{\prime}+\delta_{2} \rho & = & \delta_{2} \\
\rho(0) & = & 1 \\
\left(-\rho \rho^{\prime}+\delta_{1} U \rho\right)(1) & = & \phi
\end{array}\right.
$$




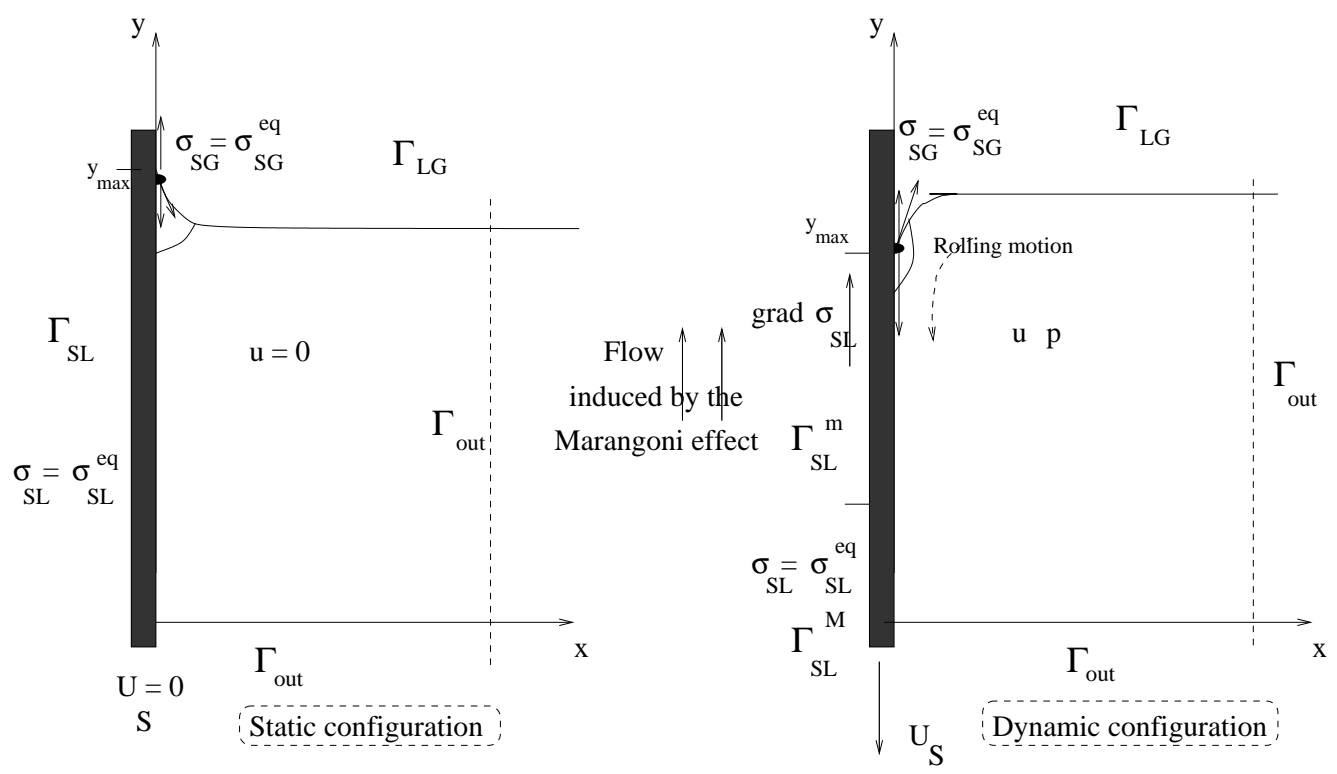

Figure 5: Plunging tape. The static and dynamic configurations (with $\left.\sigma_{L G}^{e q}>\sigma_{S L}^{e q}\right)$ 
with $\delta_{1}, \delta_{2}$ and $\phi$ defined by (24) and (25) respectively.

We set: $\sigma_{L G}^{e q}=70, \sigma_{S L}^{e q}=20$ and $\sigma_{S G}^{e q}=50 \mathrm{mN} / \mathrm{m}$.

In the static case, we have: $\cos \left(\theta_{s}\right)=\frac{\sigma_{S G}^{e q}-\sigma_{S L}^{e q}}{\sigma_{L G}^{e q}} \approx 0.429$ hence $\theta_{s} \approx$ $64.6^{\circ}$. In the dynamic case, the Young equation is supposed to remain valid and the case $\theta_{d}>90^{\circ}$ corresponds to: $\sigma_{S G}^{e q}=50<\sigma_{S L}<\sigma_{L G}^{e q}=70$.

We set $\tau^{*}=10^{-3} s$ and $U^{*}=5.10^{-2} \mathrm{~ms}^{-1}$-see [1]-. Hence, $l \approx \tau^{*} U^{*}=$ $5.10^{-5} \mathrm{~m}$.

We set $U_{S}=-1=U_{\text {stokes }}(0)$ (the no slip boundary condition value for the bulk flow) and $U(x)=\frac{1}{2}\left(U_{S}+U_{\text {stokes }}(x)\right)=\left(\frac{1}{4} x-1\right)$.

It remains to set the following two parameters: the product $\lambda . \rho^{*}$ and $\rho_{1}^{e q}$.

For the present computation we set: $\lambda \cdot \rho^{*}=10^{-6}$ and $\rho_{1}^{e q}=1 / 5$.

We obtain $\phi=-2.5$. In others respects, for obvious computational reasons, we set $l=10 \tau^{*} U^{*}$ and we obtain $\delta_{1}=25$ and $\delta_{2}=250$.

Let us notice that the state equation $\sigma_{i}=\gamma\left(\rho_{0}^{s}-\rho_{i}\right), i=1,2$, implies that $\rho_{1}^{e q}<\rho_{2}^{e q}=1$. (Recall: the indice 2 is relative to the solid-liquid interface, $\sigma_{2}=\sigma_{S L}$ ).

Let us point out that all the assumptions on data presented in the mathematical analysis section are satisfied.

The functions $\rho, \rho^{\prime}, \sigma_{S L}$ and $\sigma_{S L}^{\prime}$ obtained are presented in Fig. 4.1 and Table 2 .

\begin{tabular}{|c|c|c||c|c|}
\hline$\sigma_{S L}\left(P_{C}\right)$ & $\sigma_{S L}^{\prime}\left(P_{C}\right)$ & $\theta_{d}\left({ }^{\circ}\right)$ & $\rho\left(P_{C}\right)$ & $\left\|\rho^{\prime}\right\|_{\infty}$ \\
\hline 66.8 & $1.0110^{6}$ & 103.9 & $2.5110^{-4}$ & 175.6 \\
\hline
\end{tabular}

Table 2: Computed values for Test c.

Let us precise that the surface tension $\sigma_{S L}$ and its gradient $\sigma_{S L}^{\prime}$ are deduced from the values of $\rho, \rho^{\prime}$ and using the state equation $\sigma_{i}=\gamma\left(\rho_{0}^{s}-\right.$ $\left.\rho_{i}\right), i=1,2$. As a matter of fact, since $\sigma_{L G}^{e q}=\gamma\left(\rho_{0}^{s}-\rho_{1}^{e q}\right)$ and $\sigma_{S L}^{e q}=$ $\gamma\left(\rho_{0}^{s}-\rho^{*}\right)$, one can deduce the values of the constants $\gamma$ and $\rho_{0}^{s}$. Then, 

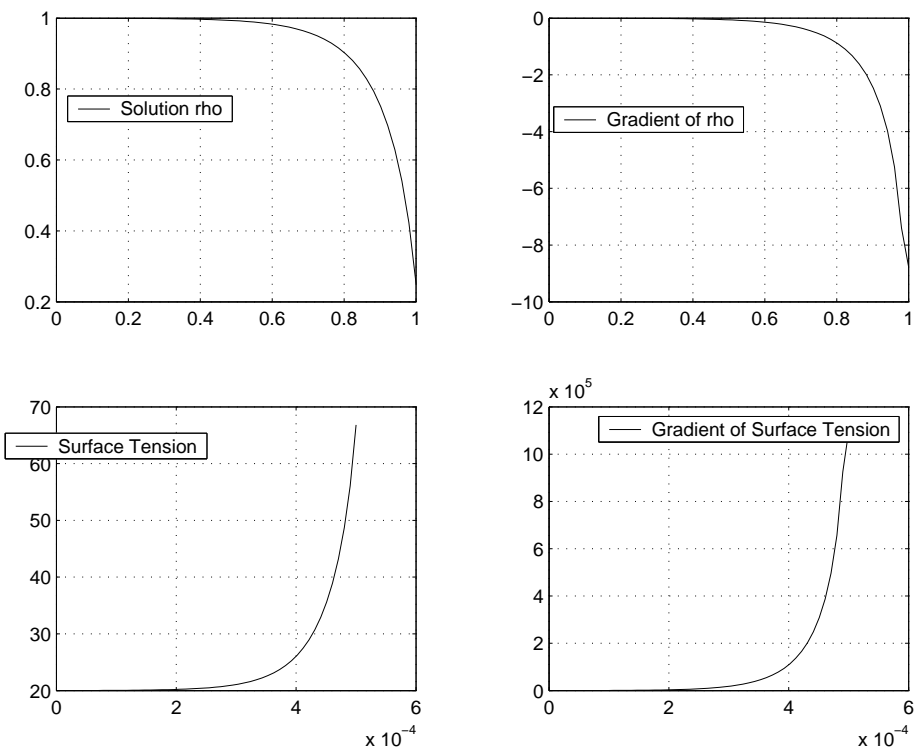

Figure 6: Test c.: $\rho, \rho^{\prime}, \sigma_{S L}$ and $\sigma_{S L}^{\prime}$ plotted

using the state equation:

$$
\sigma_{S L}(y)=\gamma\left(\rho_{0}^{s}-\rho(y)\right)
$$

we obtain the value of the surface tension coefficient $\sigma_{S L}$. Finally, we have:

$$
\theta_{d}=\cos ^{-1}\left(\frac{\sigma_{S G}^{e q}-\sigma_{S L}\left(P_{C}\right)}{\sigma_{L G}^{e q}}\right)
$$

where $P_{C}$ denotes the triple point liquid-solid-gas.

Qualitatively, this numerical result presents a variation of the surface tension $\sigma_{S L}$ and a computed dynamical wetting angle $\theta_{d}$ mechanically admissible. In other respect, let us notice that in the vicinity of the triple point $P_{C}$, the gradient $\sigma_{S L}$ is very large (a maximum amplitude of $10^{6}$ ).

The choice of the two parameters values of $\lambda \rho^{*}$ and $\rho_{1}^{e q}$ is the main uncertainty of the model. The present choice leads to an admissible surface tension $\sigma_{S L}$. Only few set of parameters we have tested, lead to a such admissible solution. 


\subsection{D bulk flow: local Marangoni effect}

Let $\theta_{d}, \sigma_{S L}, \sigma_{S L}^{\prime}$ and $\Gamma_{L G}$ be given, we compute the solution of a simplified HFSM: a Stokes model with the free surface given and fixed. Then, we can observe numerically the influence of the Marangoni source term $\sigma_{S L}^{\prime}$ on the bulk fluid motion.

The 2D bulk flow model Let us write the steady-state HFSM considered.

Gravitational forces $g$ and external pressure $p_{e x t}$ are neglected. The geometry $\Omega$ considered is presented in Fig. 4.2.

Also, we neglect the curvature effect, we set $\kappa=0$.

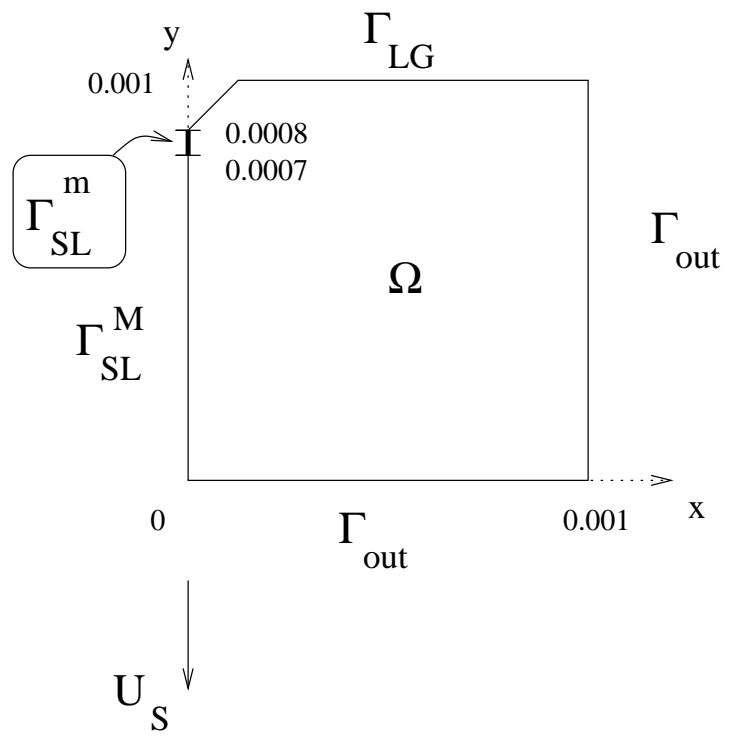

Figure 7: Geometry of the 2D case test.

The test model is the following. Given $\theta_{d}, \sigma_{S L}^{\prime}$ and $\Gamma_{L G}$, find $(u, p)$ 
satisfying:

$$
\begin{cases}-\partial_{1} \Sigma_{i 1}-\partial_{2} \Sigma_{i 2}=0 & \text { in } \Omega \quad i=1,2 \\ \operatorname{div}(u)=0 & \text { in } \Omega \\ \Sigma_{n}=\Sigma_{\tau}=0 & \text { on } \Gamma_{L G} \\ u=U_{S} & \text { on } \Gamma_{S L}^{M} \\ u . n=0 \text { and } \Sigma_{\tau}=-\beta\left(u-U_{S}\right)+\frac{1}{2} \sigma_{S L}^{\prime} & \text { on } \Gamma_{S L}^{m} \\ \Sigma_{n}=\Sigma_{\tau}=0 & \text { on } \Gamma_{\text {out }}\end{cases}
$$

with $\Sigma_{i j}=-p \delta_{i j}+\mu\left(\partial_{i} u_{j}+\partial_{j} u_{i}\right), 1 \leq i, j \leq 2, \Gamma_{S L}=\Gamma_{S L}^{M} \cup \Gamma_{S L}^{m}$ and $\partial \Omega=\Gamma_{S L} \cup \Gamma_{L G} \cup \Gamma_{\text {out }}$.

The slip coefficient $\beta>0$ is given. Typically, $\beta \approx{ }^{\mu} \overline{h_{l}} \approx \frac{10^{-3}}{10^{-8}}=10^{5}\left(h_{l}\right.$ is the layer thickness).

The only source terms of the model are $U_{S}$ and $\sigma_{S L}^{\prime}$ And, for $\sigma_{S L}^{\prime} \equiv 0$, the unique solution of $(42)$ is $(u, p)=\left(U_{S}, 0\right)$ (the pressure being defined up to a constant).

Therefore, in the numerical experiments presented below, we observe and measure directly the influence of $\sigma_{S L}^{\prime}$ on the fluid motion in the bulk. In others words, we observe the influence of the local Marangoni term on the macroscopic bulk flow.

Weak formulation For $u \in\left(H^{1}(\Omega)\right)^{2}$ satisfying (42) and for all $v \in$ $\left(H^{1}(\Omega)\right)^{2}$ we have:

$$
\sum_{i=1}^{2} \sum_{j=1}^{2} \int_{\Omega} \Sigma_{i j} \partial_{j} v_{i} d x=\int_{\partial \Omega}\left[\Sigma_{n} v . n+\Sigma_{\tau} v \cdot \tau\right] d s
$$

We set:

$$
\begin{gathered}
W_{0}=\left\{u, u \in\left(H^{1}(\Omega)\right)^{2}, u . n=0 \text { on } \Gamma_{S L}^{m}, u=0 \text { on } \Gamma_{S L}^{M}\right\} \\
W_{t}=\left\{u, u \in\left(H^{1}(\Omega)\right)^{2}, u . n=0 \text { on } \Gamma_{S L}^{m}, u=U_{S} \text { on } \Gamma_{S L}^{M}\right\}
\end{gathered}
$$

The weak formulation of the test model is

$$
\left(\mathcal{P}_{S T}\right)\left\{\begin{array}{l}
\text { Find }\left(\left(u_{1}, u_{2}\right), p\right) \in W_{t} \times L^{2}(\Omega) / \mathbb{R} \text { such that: } \\
\sum_{j=1}^{2} \int_{\Omega} \Sigma_{1 j} \partial_{j} v_{1} d x=0 \quad \forall\left(v_{1}, 0\right) \in W_{0} \\
\sum_{j=1}^{2} \int_{\Omega} \Sigma_{2 j} \partial_{j} v_{2} d x+\beta \int_{\Gamma_{S L}^{m}} u_{2} v_{2} d x=\int_{\Gamma_{S L}^{m}} g_{s i i p} v_{2} d s \quad \forall\left(0, v_{2}\right) \in W_{0} \\
\int_{\Omega} \operatorname{div}(u) q d x=0 \quad \forall q \in L^{2}(\Omega) / \mathbb{R}
\end{array}\right.
$$


with $g_{s l i p}=\left(\beta U_{S}+\frac{1}{2} \sigma_{S L}^{\prime}\right)$.

We solve $\left(\mathcal{P}_{S T}\right)$ using the Hood-Taylor finite element method (2nd order method). The pressure equation is solved using the augmented Lagrangian method and the Uzawa's algorithm. The program has been developed using Rheolef, a $\mathrm{C}++$ finite element environment ${ }^{1}$, and has been fully tested with explicit solutions.

Numerical results We set $U_{S}=\left(0,-10^{-2}\right)^{T}$ and $L=10^{-3}$ (in I.S. units). We set $\tau^{*}=10^{-3}$ hence $l \approx \tau^{*} U^{*}=10^{-5}$ and $\varepsilon \approx \frac{l}{L} \approx 10^{-2}$.

We have the Capillary number $C a=\frac{\mu U^{*}}{\sigma_{S L}} \approx 10^{-6}$ and the Reynolds number $R e=\frac{\hat{\rho} U^{*} L^{*}}{\mu} \approx 50$.

It remains to set the two following parameters: the slip coefficient $\beta$ and the given surface tension gradient $\sigma_{S L}^{\prime}$.

For both tests, we set $\beta=10^{5}$, and

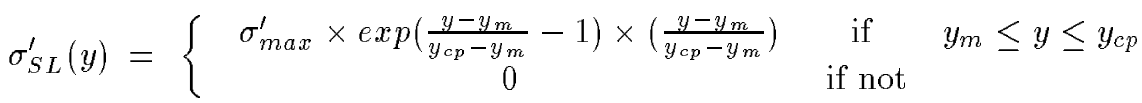

where $y_{m}=0.00075$ is the middle point of the boundary part $\Gamma_{S L}^{m}$ and $y_{c p}=0.0008$ is the contact point $\mathrm{y}$-coordinate.

Therefore, the present given function $\sigma_{S L}^{\prime}(y)$ behaves qualitatively like the computed one in Test c. (Fig. 4.1).

We present two examples. The first example with $\sigma_{\max }^{\prime}=10^{3}$ (Test d.) and the second one with $\sigma_{\max }^{\prime}=5.10^{3}$ (Test e.).

The computations have been performed on a coarse mesh and on a fine one. The fine mesh comprises 6082 elements and 3184 vertices. No particular mesh sensitivity has been noticed.

In the first example (Test d.), we observe a simple flow. The given source term $g_{s l i p}$ is strictly negative in $\Gamma_{S L}^{m}$. The computed y-coordinate velocity $u_{2}$ is strictly negative and slowed down. See figures 4.2, 4.2, 4.2, 4.2 .

In the second example (Test e.), we observe a more complex flow. The given source term $g_{\text {slip }}$ changes of sign in the vicinity of $7.810^{-4}$. The

\footnotetext{
${ }^{1} \mathrm{P}$. Saramito and N. Roquet, Rheolef C++ finite element environment, http://www-
} lmc.imag.fr/lmc-edp/Pierre.Saramito/rheolef 


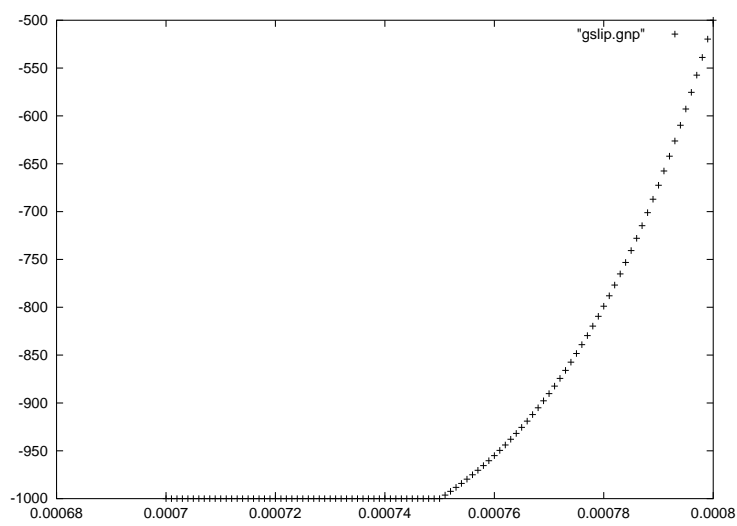

Figure 8: Test d. The local source term $g_{\text {slip }}$ on $\Gamma_{S L}^{m}$

computed y-coordinate velocity $u_{2}$ changes of sign too, in the same area. Thus, we observe a local recirculation: the Marangoni term induces a recirculation in the vicinity of the contact line. See figures 4.2, 4.2, 4.2.

Let us recall that the model is too simplified in order to interpret these numerical results in a mechanical point of view. To this end, one must take into account the free surface dynamic, the capillary forces and eventually consider the local slip boundary conditions in the upper part of the vicinity of the triple line i.e. on $\Gamma_{L G}$. Nevertheless, these numerical results show clearly the effects of the local slip boundary condition on the fluid motion in the bulk.

These numerical results are a first step for the simulation of the rolling motion and the dynamic of the contact angle using the Shikhmurzaev's model. The development of the full model (4)-(17) is under progress. The numerical schemes, algorithms and numerical results obtained will be published in an other article.

\section{References}

[1] T.D. Blake \& al., Experimental evidence of nonlocal hydrodynamic influence on the dynamic contact angle, Physics of fluids, 118 (1999) 1995-2007. 


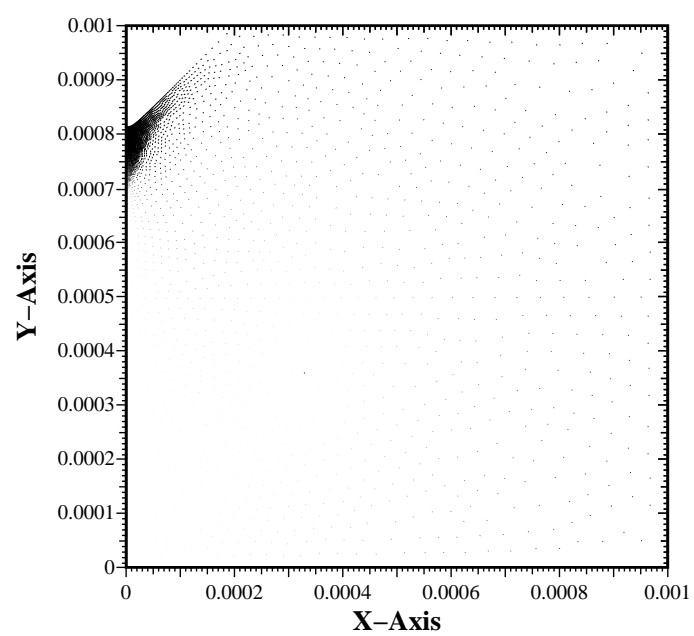

Figure 9: Test d. The vector field $\left(u-U_{s}\right)$ in $\Omega$ 


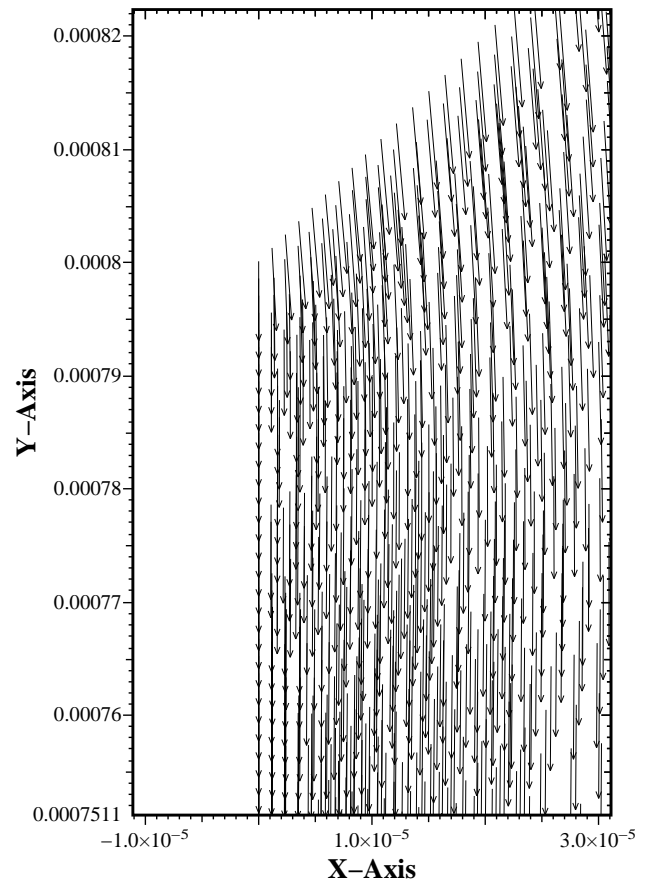

Figure 10: Test d. The velocity field $u$ near the contact point 


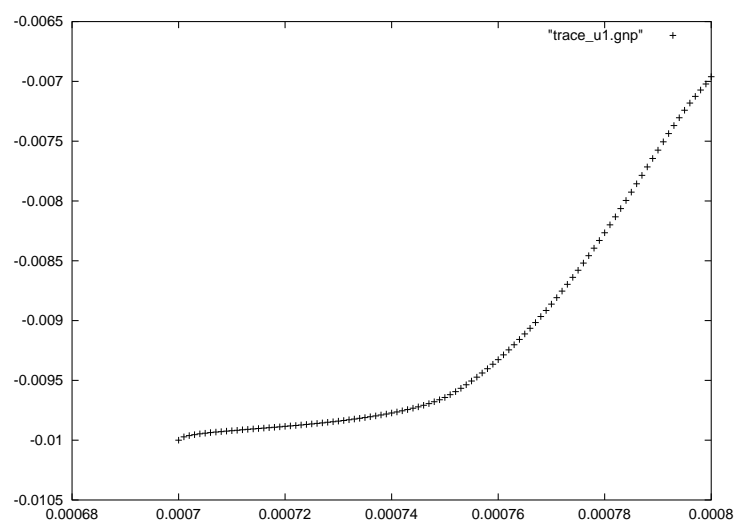

Figure 11: Test d. $u_{2}$ on $\Gamma_{S L}^{m}$

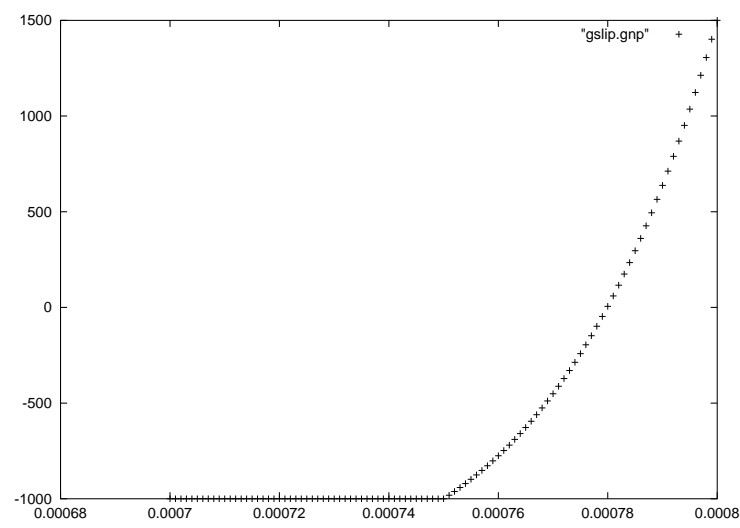

Figure 12: Test e. The local source term $g_{\text {slip }}$ on $\Gamma_{S L}^{m}$ 


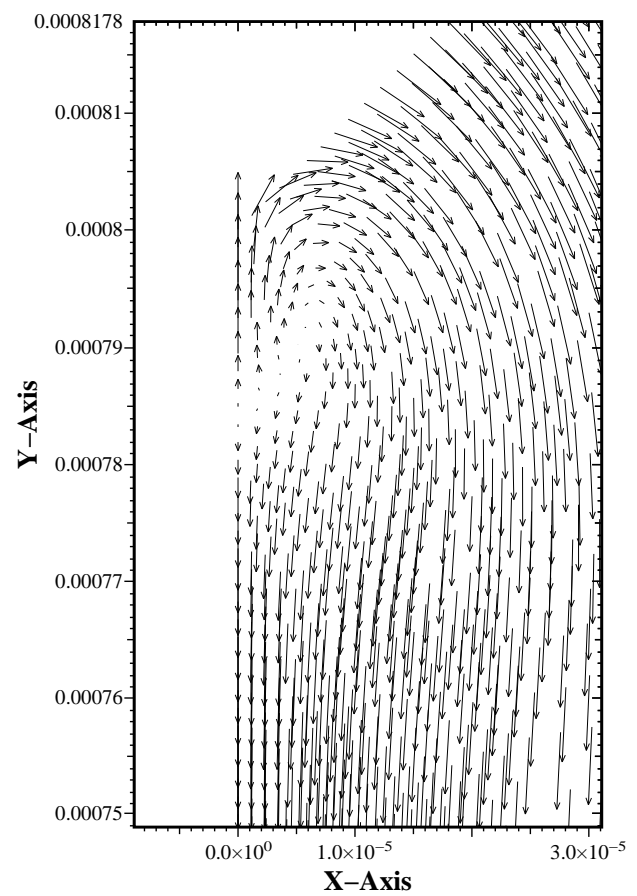

Figure 13: Test e. The velocity $u$ near the contact point 


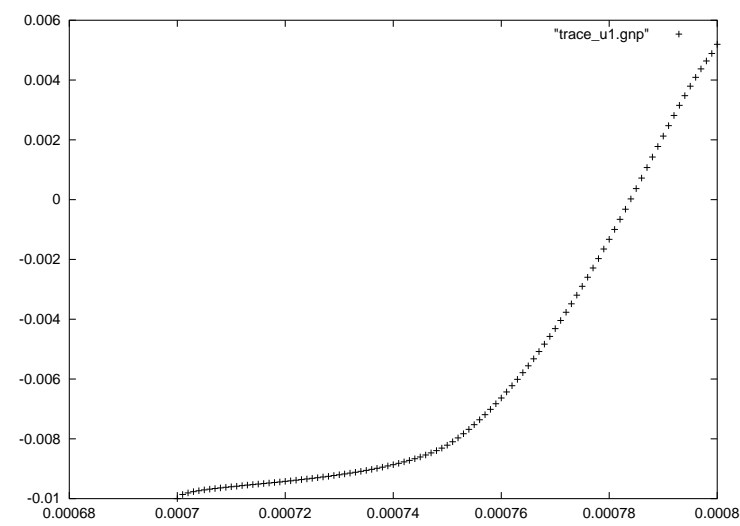

Figure 14: Test e. The trace of the velocity on $\Gamma_{S L}^{m}$

[2] G. Caloz, J. Rappaz, Numerical Analysis for Nonlinear and Bifurcation Problems, Handbook of Numerical Analysis, Vol. V, Eds P.G. Ciarlet \& J.L. Lions, 1997.

[3] R.G. Cox, The dynamics of spreading of liquids on a solid surface. Part 1. Viscous flow, J. Fluid. Mech., 168, 169-194 (1986).

[4] P.G. de Gennes, Wettings: statics and dynamics, Reviews Modern Phys., 57, 3 (1985).

[5] V.E.B. Dussan, S.H. Davis, On the motion of a fluid-fluid interface along a solid surface, J. Fluid Mech. 65, 71-95 (1974).

[6] V.E.B. Dussan, The moving contact line: the slip boundary condition, J. Fluid Mech. 77, 665-684 (1976).

[7] D. Gilbarg and N. Trudinger, Elliptic partial differential equations of second order, (Springer, 1977).

[8] L.M. Hocking, A moving fluid interface. Part II. The removal of the force singularity by a slip flow. J. Fluid Mech., 79, 209-229 (1977).

[9] Y.D. Shikhmurzaev, The moving contact line problem on a smooth solid surface, Int. J. Multiphase Flow, 19 (4) (1993) 589-610.

[10] Y.D. Shikhmurzaev, Mathematical modeling of wetting hydrodynamics, Fluid dynamics Research, 13 (1994) 45-64.

[11] Y.D. Shikhmurzaev, Moving contact lines in liquid/liquid/solid systems, J. Fluid Mech., 334 (1997) 211-249. 